\title{
Complexity of resonances exhibited by a nonlinear micromechanical gyroscope: an analytical study
}

\author{
Jan Awrejcewicz • Roman Starosta • \\ Grażyna Sypniewska-Kamińska
}

Received: 10 May 2018 / Accepted: 21 August 2018 / Published online: 29 August 2018

(C) The Author(s) 2018

\begin{abstract}
Dynamics behavior of the micromechanical gyroscope designed for measuring one component of the angular velocity is studied in the paper. The Cardan suspension is applied to connect the sensing plate with the substrate whose angular velocity is measured. The gimbal and the plate with sensors are connected via torsional joints. Vibrating motion of the sensing plate is excited mainly by a torque resulting from the Coriolis effect. The mathematical model equations have been derived using the Lagrange equation of the second kind. Both nonlinear effects of the geometrical nature and the nonlinear characteristics of the torsional joints are taken into account. The governing equations are solved with help of the method of multiple scales in time domain that belongs to the broad class of asymptotic methods. The approximate solution of analytical form has been obtained for non-resonant vibration as well as for the case of the main and internal resonances that occur simultaneously. Analytical form of solution allows for extensive analysis of the behavior of the system. The desirable state of the gyroscope work is steady-state vibration in resonance that is discussed in detail.
\end{abstract}

\footnotetext{
J. Awrejcewicz

Department of Automatics and Biomechanics, Technical University of Łódź, Lodz, Poland

R. Starosta ( $\varangle)$. G. Sypniewska-Kamińska Institute of Applied Mechanics, Poznan University of Technology, Poznan, Poland

e-mail: roman.starosta@put.poznan.pl
}

Keywords Micromechanical gyroscope - Resonances $\cdot$ Multiple scales method

\section{Introduction}

Gyroscopes are present in a broad range of engineering systems such as air vehicles, automobiles, and satellites to track their orientation and control their path. Besides the directional gyroscopes, there are variety of gyroscopes (e.g., mechanical, optical and vibrating) that are being used to measure the angular velocity. The critical part of the conventional mechanical gyroscope is a wheel spinning at a high speed. Therefore, conventional gyroscopes although accurate are bulky and very expensive and they are applicable mainly in the navigation systems of large vehicles, such as ships, airplanes, space crafts, etc.

Micromechanical gyroscopes and angular rate sensors allow for signification miniaturization in contrary to solid-state gyroscopes, laser ring and fiber optic gyroscopes.

Progress in micromachining technology embraces the development of the miniaturized gyroscopes with improved performance and low power consumption that allow the integration with electronic circuits. Their manufacturing cost is also significantly lower [1,2]. Such type of gyroscopes belongs to broad class of microelectromechanical systems (MEMS). Practically, any device fabricated using photo-lithography-based techniques with micrometer scale features that utilizes 
both electrical and mechanical functions could be considered as MEMS.

The operating principle of vibrating gyroscopes is based on the transfer of the mechanical energy among two vibrations modes via the Coriolis effect which occurs in the presence of a combination of rotational motions about two orthogonal axes. The drive mode is mainly generated employing the electrostatic actuation mechanism.

However, it is widely recognized that miniaturization achieved via fabrication technologies requires detailed studies from a point of view of nonlinear dynamical systems in order to understand and control of sometimes unexpected behavior of microcomponents, micromachines and MEMS/NEMS, and in particular of micromechanical gyroscopes. Reliable modeling of the micromechanical vibratory gyroscopes allows for improvement in sensitive elements and circuit design, and hence, it has an important impact on achieving high performances of the mentioned micromechanical systems. In other words the micro- and nanotechnologies require support of theoretical approaches based on theory of vibrations and nonlinear phenomena. In what follows, we briefly describe state of the art of the recent achievement in modeling and analysis on some chosen MEMS/NEMS and micro-/nanogyroscopes.

Tuner et al. [3] pointed out importance of parametric resonances in a micromechanical system. Lifshitz and Cross [4] investigated a response of the microring gyroscope under combined external forcing and parametric excitation to achieve required parametric amplification.

Gallacher et al. [5] proposed a control scheme for a MEMS electrostatic resonant gyroscope subjected to both harmonic forcing and parametric excitation.

Nayfeh and Younis [6] investigated dynamics of MEMS resonators under superharmonic/subharmonic excitations.

Kacem et al. [7] improved the performance of NEMS sensors based on employment of theoretical approaches of modeling nonlinear dynamics of nanomechanical beam resonators.

Lestev and Tikhonov [8] analyzed nonlinear dynamical behavior of micromechanical gyroscopes using the method of averaging. They pointed out that even though the parameters of the microstructural components are chosen in a way to provide a linear response, it cannot be achieved due to the fabrication errors. They investigated stable steady-state modes of vibra- tory micromechanical gyroscopes, and they presented the corresponding resonance curves.

Nonlinear dynamics and chaos of electrostatically actuated MEMS resonators under two-frequency external and parametric excitations were analyzed by Zhang et al. [9]. In particular, they illustrated effects of nonlinear square damping on the frequency response. Resonance frequencies and nonlinear dynamic characteristics were also reported. However, their investigation concerned relatively simple model consisting of a mass-spring-damper system.

Martynenko et al. [10] studied nonlinear phenomena of a vibrating micromechanical gyroscope with a ring resonator flexibly supported. The Krylov-Bogolubov averaging method was employed to predict fabrication errors, unstable branches of resonance curves, and quenching phenomenon.

Sang Won Yoon et al. [11] modeled vibratory ring gyroscopes by four vibration models (two flexural and two translation). The developed model consisted of the ring structure, the support-string structure, and the electrodes. It was shown that the developed model becomes vibration sensitive in the presence of both non-proportional damping and the sense electrodes capacitive nonlinearity.

Matheny et al. [12] studied nonlinear mode-coupling in nanomechanical systems. They demonstrated measurement protocol and design rules for getting accurate in situ characterization of nonlinear properties of NEMS resonators. In particular, the employment of the Euler-Bernoulli beam model was validated through the carried out laboratory measurements.

Ovchinnikova et al. [13] developed a model of micromechanical gyroscope using inertia properties of standing elastic waves providing maximum vibration amplitude with minimum control. The employed schemes of stabilization of the excited amplitude reduced nonlinear transformation characteristics. The method allowed for computation of the envelope of the fundamental mode of vibration of the governing two second-order ODEs yielded by the Bubnov-Galerkin approach.

Yoon et al. [14] studied a micromechanical vibrating ring gyroscope under high shocks based on mathematical analysis supported by the finite element method. They suggested employment of the developed vibrating ring gyroscope in navigation systems when both performance and high shock resistance are crucial in getting proper measurements. 
Lestev [15] investigated combination resonances of sensitive elements of micromechanical gyros under translational and angular motions of the platform. The governing nonlinear ODEs were derived and the obtained results were validated experimentally.

Nitzan et al. [16] considered parametric amplification of a micromechanical resonating disk gyroscope taking into account of self-induced parametric excitation and Coriolis forces. The parametric self-induced amplification was yielded by nonlinear stiffness coupling between degenerate orthogonal vibration modes in a high-quality-factor micromechanical resonator.

Defoort et al. [17] analyzed occurrence of synchronization between two degenerate resonance modes of a microdisk resonator gyroscope. The carried out consideration were based on two second-order ODEs including a geometric nonlinearity of a cubic type. They demonstrated how mutual synchronization between modes was robust over temperature variation.

An impact of a cubic nonlinearity on the operation of a rate-integrating gyroscope was studied by Nitzan et al. [18]. It was shown how below the bifurcation threshold of cubic nonlinearity a splitting of angledepending frequency between two resonant gyroscope modes occurred which impacted angle-dependent bias, quadrature error and controller efficacy. The method of compensating for angle-dependent frequency error was proposed and was experimentally validated.

A useful overview of gyroscopic technology including mechanical and optical at macro- and microscale was given by Passaro et al. [19].

In the present work, we conduct an analysis of dynamics of a MEMS gyroscope. This microdevice is a torsional resonator. Resonance is the desirable state of work of this sensor, so the elastic properties should be appropriately matched. Designing the resonator, only linear elasticity is taken into account. There arises the question what is the significance of the nonlinear properties of resilient resonator elements. Therefore, we propose the mathematical model describing motion of the MEMS gyroscope taking into account the nonlinear effects generated by the elastic properties of the suspension elements [20]. The main objective of the paper is to obtain and to examine the resonant responses of the considered system.

The paper is organized in the following way. Section 2 deals with a description of the further studied micromechanical gyroscope. Equations of motion are derived in Sect. 3. Section 4 reports the analyt- ically obtained approximate solutions to the governing two second-order nonlinear ODEs in the case of non-resonant vibrations. Resonant vibrations are studied analytically and numerically in Sect. 5. Section 6 is devoted to investigation of steady-state gyroscope responses. Concluding remarks are given in Sect. 7.

\section{Description of micromechanical gyroscope}

Dynamics of the torsional micromechanical gyroscope used to measure one component of the angular velocity is the subject of the paper. The Cardan suspension idea is applied to connect the sensing plate with the substrate whose angular velocity is measured. A diagram of the MEMS gyroscope is presented in Fig. 1. The sensing element " 3 " connects to the intermediate gyroscope part, i.e., the gimbal "2" via two torsional joints having a common axis called the sense axis. Two torsional joints link the gimbal with the anchors "1" mounted on the substrate which can be movable in the general case. These connectors are also aligned along a common straight line designating the drive axis. The gimbal is loaded by the external harmonically changing torque. The sense and drive axes around which the sensing plate and the gimbal can rotate independently are mutually orthogonal. In the system, there is the coupling effect. When the gyroscope is subjected to a rotation about $z$-axis caused by the substrate motion the sinusoidal Coriolis torque at the frequency of drive-mode oscillations is induced in the sense direction. The Coriolis torque excites the proof-mass to oscillate around the sense axis. This response, caused by the Coriolis effect

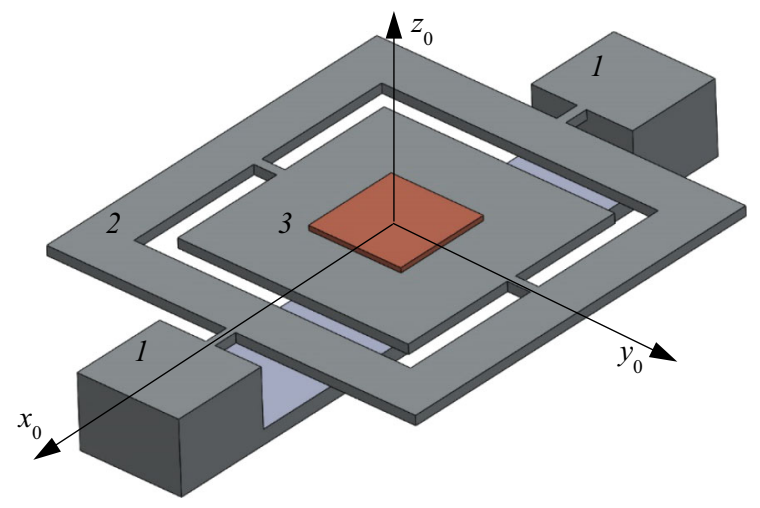

Fig. 1 Micromechanical gyroscope suspended on a set of two pivoted and mutually orthogonal pivot axes; 1-anchor, 2gimbal, 3-sensing plate 
Fig. 2 The angles of rotation $\Phi$ and $\Theta$ : a the rotation of $F_{1}$ by $\Phi$ about $x_{0}$-axis; $\mathbf{b}$ the superposition of two rotations: $F_{1}$ by $\Phi$ about $x_{0}$-axis and $F_{2}$ by $\Theta$ around $y_{1}$

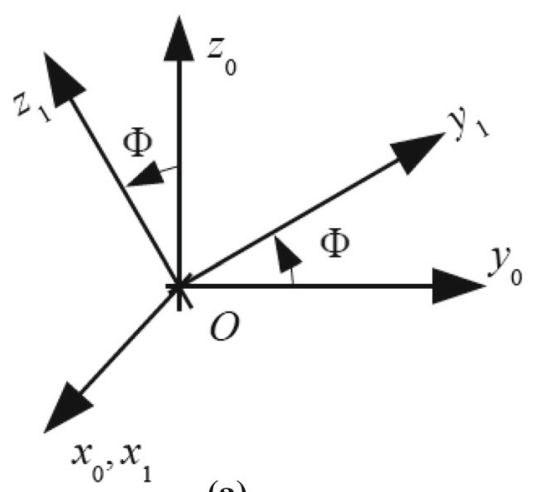

(a)

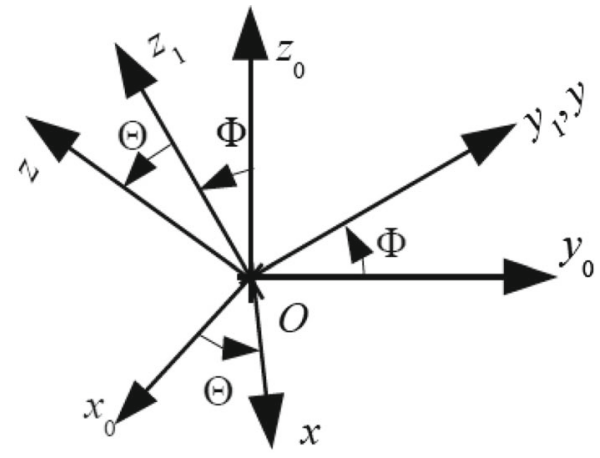

(b) and proportional to the angular velocity being measured, is registered by the detection electrodes. In order to attain the maximum response of the proof-mass, the desirable work regime of the micromechanical system is the resonance around both sense and drive axes. This state is achieved at the designing stage what especially involves the proper choice of the inertial properties of the MEMS parts and the elastic features of the torsional joints. It seems to be advisable to consider the influence of the nonlinear elastic properties of the torsional connectors on the micromechanical system operation. Including into consideration these nonlinearities can increase the working precision of the gyroscope.

Assuming the gimbal and the sensing plate are the rigid bodies, we model the torsional gyroscope as a two degrees-of-freedom (2-DOF) mass-springdamper system with one sensing axis, so it is designed to measure one coordinate of the angular velocity of the substrate.

When describing the movement of MEMS parts, it is helpful to introduce three reference frames shown in Fig. 2. These frames have the common origin at the point $O$. Point $O$ is also the center of mass of the whole gyroscope. In the frame $F_{0}$ with the Cartesian coordinate system $O x_{0} y_{0} z_{0}$ the anchors and thus also the substrate whose angular velocity is measured are motionless. The frame $F_{1}$ with the coordinate system $O x_{1} y_{1} z_{1}$ is fixed to the gimbal, whereas the frame $F$ in which it is assumed the coordinate system Oxyz is rigid connected with the sensing plate. At stable equilibrium position of the MEMS presented in Fig. 1, the axes of all these frames overlap with each other. Each of the introduced frames is non-inertial. The frame $F_{1}$ which can oscillate about the drive axis $x_{0}$ is presented in Fig. $2 \mathrm{a}$ in the position rotated by $\Phi$ counterclockwise.
In Fig. 2b, the frame $F$ oscillating around the sense axis $y_{1}$ is depicted in the position being a result of the rotation by $\Theta$, also counterclockwise, and the rotation of the frame $F_{1}$ associated to the gimbal. The anchors and the substrate can rotate about a fixed pivot axis. Let us assume that its absolute angular velocity $\boldsymbol{\Omega}_{z}$ projected on the axes of the frame $F_{0}$ is $\boldsymbol{\Omega}_{z}=\left[0,0, \Omega_{z}\right]^{T}$, where $\Omega_{z}$ is to be measured.

The absolute gimbal angular velocity $\boldsymbol{\Omega}_{1}$ is a superposition of the substrate rotation and own rotation about the drive axis. When projecting it onto the axes of the frame $F_{1}$, we obtain

$\boldsymbol{\Omega}_{1}=\left[\dot{\Phi}, \Omega_{z} \sin \Phi, \Omega_{z} \cos \Phi\right]^{T}$

The absolute angular velocity $\boldsymbol{\Omega}$ of the sensing plate written in the reference frame $F$ and being a result of the substrate motion, gimbal rotation and own rotation about $y$-axis is

$$
\begin{aligned}
\boldsymbol{\Omega}= & {\left[-\Omega_{z} \cos \Phi \sin \Theta+\cos \Theta \dot{\Phi}, \Omega_{z} \sin \Phi\right.} \\
& \left.+\dot{\Theta}, \Omega_{z} \cos \Phi \cos \Theta+\dot{\Phi} \sin \Theta\right]^{T} .
\end{aligned}
$$

\section{Equations of motion}

The considered micromechanical system has two degrees of freedom in its motion relative to the substrate. The rotation angles $\Phi(t)$ and $\Theta(t)$ are assumed to be the general coordinates. The point $O$ that is the mass center both of the sensing plate and the gimbal is constantly at rest. Due to assumed symmetry, the axes of reference frames $F_{1}$ and $F$ are the principal axes of inertia of the gimbal and the sensing plate, respectively. Therefore, the inertia tensors of each of the gyroscope 
parts related to their own principal axes have the diagonal form independently of the current system configuration. Let $I_{x}, I_{y}$ and $I_{z}$ denote moments of inertia of the gimbal about its principal inertia axes, whereas $J_{x}, J_{y}, J_{z}$ stand for the principal moments of inertia of the senor plate with respect to the axes $x, y$ and $z$. So, we can write the inertia tensors of both gyroscope parts as

$\hat{\mathbf{I}}=\operatorname{diag}\left(I_{x}, I_{y}, I_{z}\right), \quad \hat{\mathbf{J}}=\operatorname{diag}\left(J_{x}, J_{y}, J_{z}\right)$.

From the viewpoint of the absolute observer, the kinetic energy of the whole system is a sum of two bilinear forms

$T=\frac{1}{2}\left(\boldsymbol{\Omega}_{1}^{T} \cdot \hat{\mathbf{I}} \cdot \boldsymbol{\Omega}_{1}+\boldsymbol{\Omega}^{\mathbf{T}} \cdot \hat{\mathbf{J}} \cdot \boldsymbol{\Omega}\right)$,

where symbol $\cdot$ denotes the inner product.

Substituting formulas (1)-(3) into Eq. (4), we get

$$
\begin{aligned}
T= & \frac{1}{2} I_{x} \dot{\Phi}^{2}+\frac{\Omega_{z}^{2}}{2}\left(I_{z} \cos ^{2} \Phi+I_{y} \sin ^{2} \Phi\right) \\
& +\frac{J_{y}}{2}\left(\Omega_{z} \sin \Phi+\dot{\Theta}\right)^{2} \\
& +\frac{J_{x}}{2}\left(\Omega_{z} \cos \Phi \sin \Theta-\dot{\Phi} \cos \Theta\right)^{2} \\
& +\frac{J_{z}}{2}\left(\Omega_{z} \cos \Phi \cos \Theta+\dot{\Phi} \sin \Theta\right)^{2}
\end{aligned}
$$

There are assumed cubic nonlinear properties of soft type for all torsional joints. Taking into account that the mass center $O$ of the whole micromechanical system remains constantly immovable, we can write the potential energy as follows

$V=\frac{1}{2} k_{11} \Phi^{2}-\frac{1}{4} k_{12} \Phi^{4}+\frac{1}{2} k_{21} \Theta^{2}-\frac{1}{4} k_{22} \Theta^{4}$,

where $k_{11}, k_{12}$ and $k_{21}, k_{22}$ are elastic coefficients of the torsional joints, respectively, for the anchors-gimbal and gimbal-sensing plate connections.

Primary role in damping mechanism play the viscous effects of gas flow which occurs between the rotating surfaces and the immovable ones. As it was mentioned, the system is excited by the driving electrostatic torque $M_{0} \sin (P t)$ applied to the gimbal. The external loading and damping moments are introduced into motion equations as the generalized forces.

The equations of motion derived using the Lagrange equations of the second kind are as follows

$$
\begin{aligned}
& \frac{1}{2}\left(2 I_{x}+J_{x}+J_{z}+\left(J_{x}-J_{z}\right) \cos (2 \Theta)\right) \ddot{\Phi} \\
& \quad+C_{1} \dot{\Phi}+k_{11} \Phi-k_{12} \Phi^{3}+\left(J_{z}-J_{x}\right) \sin (2 \Theta) \dot{\Phi} \dot{\Theta} \\
& \quad+\frac{\Omega_{z}^{2}}{4}\left(2 I_{z}-2 I_{y}+J_{x}-2 J_{y}+J_{z}\right. \\
& \left.\quad+\left(J_{z}-J_{x}\right) \cos (2 \Theta)\right) \sin (2 \Phi) \\
& \quad-\Omega_{z} \dot{\Theta}\left(J_{y}+\left(J_{x}-J_{z}\right)\right. \\
& \quad \times \cos (2 \Theta)) \cos \Phi=M_{0} \sin (P t) \\
& J_{y} \ddot{\Theta}+C_{2} \dot{\Theta}+k_{21} \Theta-k_{22} \Theta^{3} \\
& \quad+\Omega_{z}^{2}\left(J_{z}-J_{x}\right) \cos \Theta \sin \Theta \cos ^{2} \Phi \\
& \quad+\Omega_{z} \dot{\Phi}\left(J_{y}+\left(J_{x}-J_{z}\right) \cos (2 \Theta)\right) \cos \Phi \\
& \quad-\left(J_{z}-J_{x}\right) \dot{\Phi}^{2} \cos \Theta \sin \Theta=0
\end{aligned}
$$

where $C_{1}$ and $C_{2}$ are the damping coefficients.

Expecting the elements of the system vibrate in very small ranges values of angles $\Phi$ and $\Theta$, so we carry out the linear approximation of trigonometric functions of these angles. It makes the equations of motion much simpler. It is convenient to transform the governing equations into non-dimensional form. For this purpose, we introduce the dimensionless time $\tau=t \omega_{1}$ and the following dimensionless parameters:

$$
\begin{aligned}
p & =\frac{P}{\omega_{1}}, \quad \omega_{z}=\frac{\Omega_{z}}{\omega_{1}}, \quad w=\frac{\omega_{2}}{\omega_{1}}, \quad c_{1}=\frac{C_{1}}{\left(I_{x}+J_{x}\right) \omega_{1}}, \\
c_{2} & =\frac{C_{2}}{J_{y} \omega_{1}}, \quad \alpha_{1}=\frac{k_{12}}{\left(I_{x}+J_{x}\right) \omega_{1}^{2}}, \quad \alpha_{2}=\frac{k_{22}}{J_{y} \omega_{1}^{2}}, \\
j_{1} & =\frac{I_{z}+J_{z}-I_{y}-J_{y}}{I_{x}+J_{x}}, \quad j_{2}=\frac{J_{z}-J_{x}}{I_{x}+J_{x}}, \\
j_{3} & =\frac{J_{y}}{I_{x}+J_{x}}, \quad j_{4}=\frac{J_{z}-J_{x}}{J_{y}}, \quad f_{0}=\frac{M_{0}}{\left(I_{x}+J_{x}\right) \omega_{1}^{2}},
\end{aligned}
$$

where $\omega_{1}=\sqrt{\frac{k_{11}}{I_{x}+J_{x}}}, \omega_{2}=\sqrt{\frac{k_{21}}{J_{y}}}$.

The governing equations take the following dimensionless form

$$
\begin{aligned}
& \ddot{\varphi}+\left(1+j_{1} \omega_{z}^{2}\right) \varphi-\alpha_{1} \varphi^{3}+\left(c_{1}+2 j_{2} \vartheta \dot{\vartheta}\right) \dot{\varphi} \\
& \quad+\left(j_{2}-j_{3}\right) \omega_{z} \dot{\vartheta}-f_{0} \sin (p \tau)=0, \\
& \ddot{\vartheta}+\left(w^{2}+j_{4} \omega_{z}^{2}-j_{4} \dot{\varphi}^{2}\right) \vartheta-\alpha_{2} \vartheta^{3} \\
& \quad+c_{2} \dot{\vartheta}+\left(1-j_{4}\right) \omega_{z} \dot{\varphi}=0,
\end{aligned}
$$

where $\varphi(\tau), \vartheta(\tau)$ correspond to the dimensional general coordinates $\Phi(t)$ and $\Theta(t)$ and are functions of the non-dimensional time. Hereafter, the dots over symbols denote derivatives respecting to dimensionless time $\tau$.

Equations (10)-(11) are supplemented with the proper initial conditions 
$\varphi(0)=\varphi_{0}, \quad \dot{\varphi}(0)=\omega_{0}, \quad \vartheta(0)=\vartheta_{0}, \quad \dot{\vartheta}(0)=\omega_{\vartheta 0}$,

where $\varphi_{0}, \omega_{\varphi 0}, \quad \vartheta_{0}, \quad \omega_{\vartheta 0}$ being known numbers describe the initial kinematic state of the gyroscope.

\section{Approximate analytical solution for non-resonant case}

The approximate analytical solution of the initial value problem (10)-(12) is obtained using multiple scales method (MSM) [21]. In accordance with MSM, the system evolution in time is described using several variables of time nature. These variables are related to each other by the so-called small parameter $\varepsilon$. We introduce three time variables in the following manner: $\tau_{0}=\tau$ is the "fast" time, whereas $\tau_{1}=\varepsilon \tau$ and $\tau_{2}=\varepsilon^{2} \tau$ play role of the "slow" times. Automatically, all functions dependent on time become the functions of the new time variables, and derivatives with respect to the original time $\tau$ are replaced by the following partial differential operators

$$
\begin{aligned}
\frac{\mathrm{d}}{\mathrm{d} \tau}= & \frac{\partial}{\partial \tau_{0}}+\varepsilon \frac{\partial}{\partial \tau_{1}}+\varepsilon^{2} \frac{\partial}{\partial \tau_{2}}, \\
\frac{\mathrm{d}^{2}}{\mathrm{~d} \tau^{2}}= & \frac{\partial^{2}}{\partial \tau_{0}^{2}}+2 \varepsilon \frac{\partial^{2}}{\partial \tau_{0} \partial \tau_{1}} \\
& +\varepsilon^{2}\left(\frac{\partial^{2}}{\partial \tau_{1}^{2}}+2 \frac{\partial^{2}}{\partial \tau_{0} \partial \tau_{2}}\right)+O\left(\varepsilon^{4}\right) .
\end{aligned}
$$

The solution of the initial value problem (10)-(12) is sought in the form of the power series of the small parameter $\varepsilon$

$$
\begin{aligned}
& \varphi(\tau ; \varepsilon)=\sum_{k=1}^{k=3} \varepsilon^{k} \phi_{k}\left(\tau_{0}, \tau_{1}, \tau_{2}\right)+O\left(\varepsilon^{4}\right), \\
& \vartheta(\tau ; \varepsilon)=\sum_{k=1}^{k=3} \varepsilon^{k} \theta_{k}\left(\tau_{0}, \tau_{1}, \tau_{2}\right)+O\left(\varepsilon^{4}\right) .
\end{aligned}
$$

Moreover, several parameters describing the micromechanical system and its loading are assumed to be small, what using the small parameter $\varepsilon$ can be written as follows

$$
c_{1}=\tilde{c}_{1} \varepsilon^{2}, \quad c_{2}=\tilde{c}_{2} \varepsilon^{2}, \quad \omega_{z}=\tilde{\omega}_{z} \varepsilon \quad f_{0}=\tilde{f}_{0} \varepsilon^{3} .
$$

When making assumptions (15), we take into account that the substrate angular velocity is much smaller than the frequencies of the movable gyroscope elements.

Relations (13)-(16) are then substituted into equations of motion (10)-(11). As a result, in the equations the small parameter $\varepsilon$ appears in the different powers. It is required each of equations to be satisfied for any value of $\varepsilon$. After arranging the components of both equations according to the powers of the small parameter, this requirement is realized by equating to zero all coefficients standing at the succeeding powers of $\varepsilon$. Then, the obtained system of equations is solved recursively [22-24].

At every step of the solving process, the secular terms have to be removed. In this way, arise an initial value problem which is associated with the basic equations set. Solution of this problem, which often is named the modulation problem, present the slow evolution of vibration amplitudes and phases in time. The essential aspects regarding the recursive solving is presented in more detail in Sect. 5 where the resonant solution is sought.

The approximate solution of initial value problem (10)-(12) which is obtained using MSM has the following form

$$
\begin{aligned}
\varphi= & a_{1} \cos \left(\tau+\psi_{1}\right)-\frac{1}{32} \alpha_{1} a_{1}^{3} \cos \left(3 \tau+3 \psi_{1}\right) \\
& +\frac{j_{2} a_{1} a_{2}^{2} \cos \left(\tau-2 w \tau+\psi_{1}-2 \psi_{2}\right)}{8(w-1)} \\
& -\frac{j_{2} a_{1} a_{2}^{2} \cos \left(\tau+2 w \tau+\psi_{1}+2 \psi_{2}\right)}{8(w+1)} \\
& +\frac{\left(j_{3}-j_{2}\right) w \omega_{z} a_{2} \sin \left(w \tau+\psi_{2}\right)}{w^{2}-1}+\frac{f_{1} \sin (p \tau)}{1-p^{2}}, \\
\vartheta= & a_{2} \cos \left(\tau+\psi_{2}\right)-\frac{j_{4} a_{1}^{2} a_{2} \cos \left(2 \tau-w \tau+2 \psi_{1}-\psi_{2}\right)}{16(w-1)} \\
& +\frac{j_{4} a_{1}^{2} a_{2} \cos \left(2 \tau+w \tau+2 \psi_{1}+\psi_{2}\right)}{16(w+1)} \\
& -\frac{\alpha_{2} a_{2}^{3} \cos \left(3 w \tau+3 \psi_{2}\right)}{32 w^{2}}-\frac{\left(j_{4}-1\right) \omega_{z} a_{1} \sin \left(\tau+\psi_{1}\right)}{w^{2}-1} .
\end{aligned}
$$

The functions $a_{1}(\tau), a_{2}(\tau), \psi_{1}(\tau), \psi_{2}(\tau)$ are solutions of the modulation problem and have form

$$
\begin{aligned}
a_{1}= & a_{10} \exp \left(-\frac{c_{1} \tau}{2}\right), \\
\psi_{1}= & \psi_{10}-\frac{3 a_{10}^{2}\left(1-\exp \left(-c_{1} \tau\right)\right) \alpha_{1}}{8 c_{1}} \\
& +\frac{1}{2}\left(j_{1}-\frac{\left(j_{2}-j_{3}\right)\left(j_{4}-1\right)}{w^{2}-1}\right) \tau \omega_{z}^{2},
\end{aligned}
$$




$$
\begin{aligned}
a_{2}= & a_{20} \exp \left(-\frac{c_{2} \tau}{2}\right) \\
\psi_{2}= & \psi_{20}-\frac{a_{10}^{2}\left(1-\exp \left(-c_{1} \tau\right)\right) j_{4}}{4 c_{1} w} \\
& -\frac{3 a_{20}^{2}\left(1-\exp \left(-c_{2} \tau\right)\right) \alpha_{2}}{8 c_{2} w} \\
& +\frac{\left(j_{3}-j_{2}\right) w^{2}+j_{4}\left(\left(1+j_{2}-j_{3}\right) w^{2}-1\right)}{2 w\left(w^{2}-1\right)} \tau \omega_{z}^{2}
\end{aligned}
$$

where initial values of the amplitudes and the phases $a_{10}, a_{20}, \psi_{10}, \psi_{20}$ are known and compatible with the initial values $\varphi_{0}, \omega_{\varphi 0}, \vartheta_{0}, \omega_{\vartheta 0}$ occurring in conditions (12).

It is worth to emphasize that the solution although approximated have however an analytical form. It describes the forced vibration of the mechanical gyroscope caused by the harmonic torque acting about the drive axis. Excluding the case when $w=0$, the solution given by formulas (17)-(22) fails when $p=1$ or $w=1$ because some denominators are then equal to zero. That are cases of main and internal resonances, respectively. These two cases determine the zone of the main and internal resonance, respectively. Therefore, solution (17)-(22) is useful to describe the gyroscope behavior in conditions being far from the resonance. The time histories of the generalized coordinates $\varphi$ and $\vartheta$ given by (17)-(18) together with (19)-(22) are presented in Fig. 3. The calculations were carried out for the following fixed values of parameters (several values of the parameters are taken from the paper [25])

$p=0.021, \quad w=0.8, \quad f_{0}=0.0043662, \quad \alpha_{1}=2$,

$\alpha_{2}=2, \quad c_{1}=0.0000575531, \quad c_{2}=0.0000575531$,

$\omega_{z}=0.0000575531, j_{1}=0, \quad j_{2}=0, \quad j_{3}=1, \quad j_{4}=0$,

$a_{10}=0.009, \quad a_{20}=0.009, \quad \psi_{10}=0, \quad \psi_{20}=0$.

In Fig. 3 the beginning of the motion of both gyroscope parts is presented. We can observe slow modula- tion of the gimbal oscillations due to external loading. In fact, on each of these two graphs are depicted two curves representing the approximate solution of initial value problem (10)-(12). One of them present the solution obtained analytically according to (17)-(22), and the second the solution obtained numerically. The difference between these curves is unnoticeable.

The accuracy evaluation of the approximate solution is estimated using the measures

$$
\begin{aligned}
& \delta_{1}=\frac{1}{\tau_{\max }} \int_{0}^{\tau_{\max }}\left|G_{1}\left(\varphi_{a}, \vartheta_{a}\right)\right| \mathrm{d} \tau, \\
& \delta_{2}=\frac{1}{\tau_{\max }} \int_{0}^{\tau_{\max }}\left|G_{2}\left(\varphi_{a}, \vartheta_{a}\right)\right| \mathrm{d} \tau,
\end{aligned}
$$

where $G_{1}\left(\varphi_{a}, \vartheta_{a}\right)$ and $G_{2}\left(\varphi_{a}, \vartheta_{a}\right)$ stand for the differential operators, i.e., the left sides of motion equations (10)-(11), $\varphi_{a}, \vartheta_{a}$ are approximate solutions obtained using MMS or numerically, and $\tau_{\max }$ is the total time. Proposed measures (23) evaluate the error of fulfillment of the governing equations of the simulation duration. The functions $\varphi_{a}, \vartheta_{a}$, irrespective of the way of their obtainment, satisfy the motion equations (10)(11) only approximately.

For the approximate solution presented in Fig. 3 and obtained using MSM, the values of error are

$\delta_{1}=6.414247 \cdot 10^{-7}, \quad \delta_{2}=3.371118 \cdot 10^{-9}$.

The values of error of the fulfillment of the governing equations by the approximate solutions obtained numerically using NDSolve method implemented in Mathematica 11.1 are

$\delta_{1}=2.562360 \cdot 10^{-7}, \quad \delta_{2}=6.434821 \cdot 10^{-8}$.

The solutions obtained using MSM satisfy the second of the motion equations with a significantly smaller error.
Fig. 3 Time histories of the forced vibration in non-resonant conditions
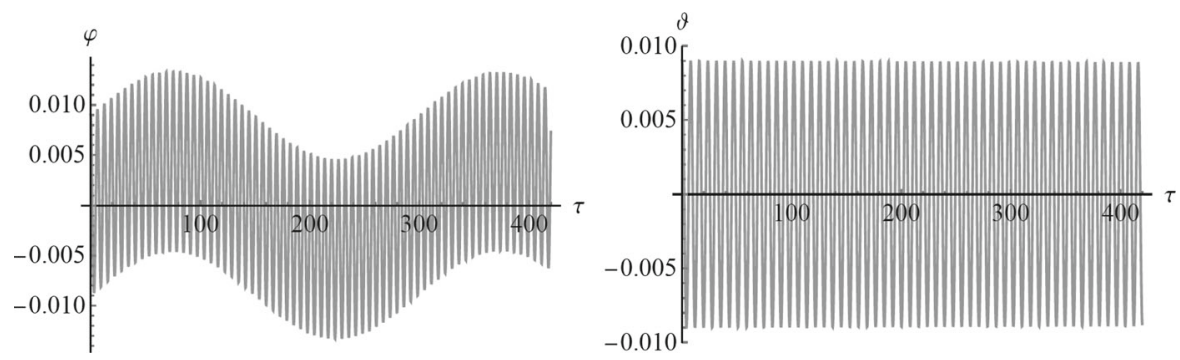


\section{Resonant vibration}

As it appears from equations (17)-(22), the frequencies of main and internal resonances are equal to each other. This characteristic feature of considered type of MEMS gyroscope results from its structure and geometry. Using non-dimensional parameters, we can write that resonances occur when $p \approx 1$ and $w \approx 1$. In view of assumptions (16), the substrate angular velocity do not affect significantly the resonance frequencies. Desirable work regime of the micromechanical system is the simultaneous resonance around both sense and drive axes. In order to achieve the state of coincidence of main and internal resonances, the system should be designed like that the both eigenfrequencies to be equal.

Assuming that the permanent equalizing the eigenfrequencies at the design stage is unobtainable in the nonlinear systems, we take into account the following resonance conditions

$p=1+\sigma_{1}, \quad w=1+\sigma_{2}$,

where $\sigma_{1}$ and $\sigma_{2}$ play role of the detuning parameters. Additionally, we assume that $\sigma_{1}=\tilde{\sigma}_{1} \varepsilon, \sigma_{2}=\tilde{\sigma}_{2} \varepsilon$.

In order to solve the initial value problem near resonance, assumptions (26) and (16) are introduced into governing equations (10)-(11). Approximate solution is determined using MSM. We introduce three time variables. The "fast" time $\tau_{0}=\tau$, and the "slow" times $\tau_{1}=\varepsilon \tau$ and $\tau_{2}=\varepsilon^{2} \tau$ replace the original time $\tau$. According to MSM rules, we employ formulas (13)(15) into motion equations (10)-(11), yielding appearance of the small parameter $\varepsilon$ in various powers. After arranging the equations with respect to the powers of the small parameter, we realize the demand the motion equations to be satisfied for any value of $\varepsilon$. We get a system of equations which have to be satisfied in order to guarantee satisfaction to the original equations. They are as follows:

- the equations of the first-order approximation

$$
\begin{aligned}
& \frac{\partial^{2} \phi_{1}}{\partial \tau_{0}^{2}}+\phi_{1}=0, \\
& \frac{\partial^{2} \theta_{1}}{\partial \tau_{0}^{2}}+\theta_{1}=0,
\end{aligned}
$$

- the equations of the second-order approximation

$$
\frac{\partial^{2} \phi_{2}}{\partial \tau_{0}^{2}}+\phi_{2}=\tilde{\omega}_{z}\left(j_{3}-j_{2}\right) \frac{\partial \theta_{1}}{\partial \tau_{0}}-2 \frac{\partial^{2} \phi_{1}}{\partial \tau_{0} \partial \tau_{1}},
$$

$$
\frac{\partial^{2} \theta_{2}}{\partial \tau_{0}^{2}}+\theta_{2}=-2 \tilde{\sigma}_{2} \theta_{1}+\tilde{\omega}_{z}\left(j_{4}-1\right) \frac{\partial \phi_{1}}{\partial \tau_{0}}-2 \frac{\partial^{2} \theta_{1}}{\partial \tau_{0} \partial \tau_{1}},
$$

- the equations of the third-order approximation

$$
\begin{aligned}
\frac{\partial^{2} \phi_{3}}{\partial \tau_{0}^{2}}+\phi_{3}= & f_{0} \sin \left(\tau_{0}+\varepsilon \tau_{0} \tilde{\sigma}_{1}\right)-j_{1} \tilde{\omega}_{z}^{2} \phi_{1} \\
& +\alpha_{1} \phi_{1}^{3}+\left(j_{3}-j_{2}\right) \tilde{\omega}_{z}\left(\frac{\partial \theta_{1}}{\partial \tau_{1}}+\frac{\partial \theta_{2}}{\partial \tau_{0}}\right) \\
& -\tilde{c}_{1} \frac{\partial \phi_{1}}{\partial \tau_{0}}-\frac{\partial^{2} \phi_{1}}{\partial \tau_{1}^{2}}-2 j_{2} \theta_{1} \frac{\partial \theta_{1}}{\partial \tau_{0}} \frac{\partial \phi_{1}}{\partial \tau_{0}} \\
& -2 \frac{\partial^{2} \phi_{1}}{\partial \tau_{0} \partial \tau_{2}}-2 \frac{\partial^{2} \phi_{2}}{\partial \tau_{0} \partial \tau_{1}}, \\
\frac{\partial^{2} \theta_{3}}{\partial \tau_{0}^{2}}+\theta_{3}= & -\left(\tilde{\sigma}_{2}^{2}+j_{4} \tilde{\omega}_{z}^{2}\right) \theta_{1}+\alpha_{2} \theta_{1}^{3} \\
& -2 \tilde{\sigma}_{2}^{2} \theta_{2}+\left(j_{4}-1\right) \tilde{\omega}_{z}\left(\frac{\partial \phi_{1}}{\partial \tau_{1}}+\frac{\partial \phi_{2}}{\partial \tau_{0}}\right) \\
& -\tilde{c}_{2} \frac{\partial \theta_{1}}{\partial \tau_{0}}-\frac{\partial^{2} \theta_{1}}{\partial \tau_{1}^{2}}+j_{4} \theta_{1}\left(\frac{\partial \phi_{1}}{\partial \tau_{0}}\right)^{2} \\
& -2 \frac{\partial^{2} \theta_{1}}{\partial \tau_{0} \partial \tau_{2}}-2 \frac{\partial^{2} \theta_{2}}{\partial \tau_{0} \partial \tau_{1}} .
\end{aligned}
$$

The solution to equations (27)-(28) is as follows

$$
\begin{aligned}
& \phi_{1}=B_{1}\left(\tau_{1}, \tau_{2}\right) \exp \left(i \tau_{0}\right)+\bar{B}_{1}\left(\tau_{1}, \tau_{2}\right) \exp \left(-i \tau_{0}\right), \\
& \theta_{1}=B_{2}\left(\tau_{1}, \tau_{2}\right) \exp \left(i \tau_{0}\right)+\bar{B}_{2}\left(\tau_{1}, \tau_{2}\right) \exp \left(-i \tau_{0}\right),
\end{aligned}
$$

where $B_{1}\left(\tau_{1}, \tau_{2}\right), B_{2}\left(\tau_{1}, \tau_{2}\right)$ are unknown complexvalued functions of slow time scales, and i denotes the imaginary unit.

The equations system (27)-(32) are solved recursively, i.e., solutions (33)-(34) are substituted into equations (29)-(30), then their solution into equations (31)-(32). Linear differential operators of the equations system (27)-(32) are the same on each level of approximation. Therefore, it is inevitable that among solutions of equations (29)-(32) the secular terms appear. In vibration case, both generalized coordinates have to be bounded; hence, all secular terms should be eliminated from each of equations (29)-(32) after introducing into them the solutions of equations of lower levels approximation.

After introducing solutions (33)-(34) into equations (29)-(30) and rejection of the secular terms, one gets

$$
\frac{\partial^{2} \phi_{2}}{\partial \tau_{0}^{2}}+\phi_{2}=0
$$




$$
\frac{\partial^{2} \theta_{2}}{\partial \tau_{0}^{2}}+\theta_{2}=0 .
$$

The general solutions of homogeneous equations (35)-(36) are unknown functions of slow time variables like in the case of solutions (33)-(34). So, it is possible to omit these solution without lost the generality. The particular solutions are obviously equal to zero and do not induce any secular terms.

Inserting solutions (33)-(34) into equations (31)(32) and elimination of the secular terms leads to the following equations

$$
\begin{aligned}
\frac{\partial^{2} \phi_{3}}{\partial \tau_{0}^{2}}+\phi_{3}= & \left(\alpha_{1} B_{1}^{2}+2 j_{2} B_{2}^{2}\right) B_{1} \exp \left(3 i \tau_{0}\right) \\
& +\left(\alpha_{1} \bar{B}_{1}^{2}+2 j_{2} \bar{B}_{2}^{2}\right) \bar{B}_{1} \exp \left(-3 i \tau_{0}\right) \\
\frac{\partial^{2} \theta_{3}}{\partial \tau_{0}^{2}}+\theta_{3}= & \left(\alpha_{2} B_{2}^{2}-j_{4} B_{1}^{2}\right) B_{2} \exp \left(3 i \tau_{0}\right) \\
& +\left(\alpha_{2} \bar{B}_{2}^{2}-j_{4} \bar{B}_{1}^{2}\right) \bar{B}_{2} \exp \left(-3 i \tau_{0}\right)
\end{aligned}
$$

Without detracting from generality, we omit the general solutions of equations (37)-(38). The particular solutions are

$$
\begin{aligned}
& \phi_{3}=-\frac{1}{8}\left(\alpha_{1} B_{1}^{2}+2 j_{2} B_{2}^{2}\right) B_{1} \exp \left(3 i \tau_{0}\right) \\
&-\frac{1}{8}\left(\alpha_{1} \bar{B}_{1}^{2}+2 j_{2} \bar{B}_{2}^{2}\right) \bar{B}_{1} \exp \left(-3 i \tau_{0}\right), \\
& \frac{\partial^{2} \theta_{3}}{\partial \tau_{0}^{2}}+\theta_{3}=-\frac{1}{8}\left(\alpha_{2} B_{2}^{2}-j_{4} B_{1}^{2}\right) B_{2} \exp \left(3 i \tau_{0}\right) \\
&-\frac{1}{8}\left(\alpha_{2} \bar{B}_{2}^{2}-j_{4} \bar{B}_{1}^{2}\right) \bar{B}_{2} \exp \left(-3 i \tau_{0}\right) .
\end{aligned}
$$

The solutions of the recursive system contain two unknown complex-valued functions $B_{1}\left(\tau_{1}, \tau_{2}\right)$, $B_{2}\left(\tau_{1}, \tau_{2}\right)$ and their complex conjugates $\bar{B}_{1}\left(\tau_{1}, \tau_{2}\right)$, $\bar{B}_{2}\left(\tau_{1}, \tau_{2}\right)$.

Elimination of the secular terms in the process of solving equation system (31)-(34) results in getting the so-called solvability conditions

$$
\begin{aligned}
& i\left(j_{3}-j_{2}\right) \tilde{\omega}_{z} B_{2}-2 i \frac{\partial B_{1}}{\partial \tau_{1}}=0, \\
& -i\left(j_{3}-j_{2}\right) \tilde{\omega}_{z} \bar{B}_{2}+2 i \frac{\partial \bar{B}_{1}}{\partial \tau_{1}}=0, \\
& i\left(j_{4}-1\right) \tilde{\omega}_{z} B_{1}-2 \tilde{\sigma}_{2} B_{2}-2 i \frac{\partial B_{2}}{\partial \tau_{1}}=0,
\end{aligned}
$$

$$
\begin{aligned}
& -i\left(j_{4}-1\right) \tilde{\omega}_{z} \bar{B}_{1}-2 \tilde{\sigma}_{2} \bar{B}_{2}+2 i \frac{\partial \bar{B}_{2}}{\partial \tau_{1}}=0 \\
& -\frac{1}{2} i \tilde{f}_{0} \exp \left(i \varepsilon \tilde{\sigma}_{1} \tau_{0}\right)+3 \alpha_{1} B_{1}^{2} \bar{B}_{1} \\
& +\frac{B_{1}}{4}\left(-4 i \tilde{c}_{1}+\left(j_{2}-j_{3}-4 j_{1}-j_{2} j_{4}+j_{3} j_{4}\right) \tilde{\omega}_{z}^{2}\right) \\
& -2 j_{2} \bar{B}_{1} B_{2}^{2}-\frac{i}{2}\left(\left(j_{2}-j_{3}\right) \tilde{\sigma}_{2} \tilde{\omega}_{z} B_{2}+4 \frac{\partial B_{1}}{\partial \tau_{2}}\right)=0
\end{aligned}
$$

$$
\begin{aligned}
& \frac{1}{2} i \tilde{f}_{0} \exp \left(-i \varepsilon \tilde{\sigma}_{1} \tau_{0}\right)+3 \alpha_{1} \bar{B}_{1}^{2} B_{1}+\frac{\bar{B}_{1}}{4} \\
& \quad \times\left(4 i \tilde{c}_{1}+\left(j_{2}-j_{3}-4 j_{1}-j_{2} j_{4}+j_{3} j_{4}\right) \tilde{\omega}_{z}^{2}\right) \\
& \quad-2 j_{2} B_{1} \bar{B}_{2}^{2}+\frac{i}{2}\left(\left(j_{2}-j_{3}\right) \tilde{\sigma}_{2} \tilde{\omega}_{z} \bar{B}_{2}+4 \frac{\partial \bar{B}_{1}}{\partial \tau_{2}}\right)=0
\end{aligned}
$$

$$
\begin{aligned}
& \left(3 \alpha_{2} B_{2}^{2}-j_{4} B_{1}^{2}\right) \bar{B}_{2}+\frac{B_{2}}{4} \\
& \times\left(-4 i \tilde{c}_{2}+\left(j_{2}+j_{3}\left(j_{4}-1\right)-4 j_{4}-j_{2} j_{4}\right) \tilde{\omega}_{z}^{2}\right) \\
& -2 i \frac{\partial B_{2}}{\partial \tau_{2}}+2 j_{4} B_{1} B_{2} \bar{B}_{1}-\frac{i}{2}\left(j_{4}-1\right) \tilde{\sigma}_{2} \tilde{\omega}_{z} B_{1}=0,
\end{aligned}
$$

$$
\begin{aligned}
& \left(3 \alpha_{2} \bar{B}_{2}^{2}-j_{4} \bar{B}_{1}^{2}\right) B_{2}+\frac{\bar{B}_{2}}{4}\left(4 i \tilde{c}_{2}+\left(j_{2}+j_{3}\left(j_{4}-1\right)\right.\right. \\
& \left.\left.-4 j_{4}-j_{2} j_{4}\right) \tilde{\omega}_{z}^{2}\right)+2 i \frac{\partial \bar{B}_{2}}{\partial \tau_{2}} \\
& +2 j_{4} B_{1} \bar{B}_{2} \bar{B}_{1}+\frac{i}{2}\left(j_{4}-1\right) \tilde{\sigma}_{2} \tilde{\omega}_{z} \bar{B}_{1}=0
\end{aligned}
$$

The solvability conditions create the system of eight partial differential equations of the first order with unknown functions $B_{1}\left(\tau_{1}, \tau_{2}\right), B_{2}\left(\tau_{1}, \tau_{2}\right), \bar{B}_{1}\left(\tau_{1}, \tau_{2}\right)$, $\bar{B}_{2}\left(\tau_{1}, \tau_{2}\right)$. It is convenient to represent these functions using the following exponential representation

$$
\begin{array}{ll}
B_{1}=\tilde{a}_{1} \exp \left(i \psi_{1}\right), & \bar{B}_{1}=\tilde{a}_{1} \exp \left(-i \psi_{1}\right), \\
B_{2}=\tilde{a}_{2} \exp \left(i \psi_{2}\right), & \bar{B}_{2}=\tilde{a}_{2} \exp \left(-i \psi_{2}\right),
\end{array}
$$

where $\tilde{a}_{1}\left(\tau_{1}, \tau_{2}\right), \quad \tilde{a}_{1}\left(\tau_{1}, \tau_{2}\right), \quad \psi_{1}\left(\tau_{1}, \tau_{2}\right), \quad \psi_{2}\left(\tau_{1}, \tau_{2}\right)$ are real-valued functions.

After changing variables in (41)-(48) according to relationships (49), we get the system of equations containing the first-order partial derivatives of unknown functions $\tilde{a}_{1}\left(\tau_{1}, \tau_{2}\right), \quad \tilde{a}_{1}\left(\tau_{1}, \tau_{2}\right), \quad \psi_{1}\left(\tau_{1}, \tau_{2}\right), \quad \psi_{2}$ $\left(\tau_{1}, \tau_{2}\right)$. We solve it with respect to these derivatives, and then insert the solutions into Eq. (13) what make possible to transform the system of partial differential equations (41)-(48) onto equivalent system of four 
ordinary differential equations. Applying inversely assumptions (16), one gets

$$
\begin{aligned}
\dot{a}_{1}=- & \frac{f_{0}}{2} \cos \left(\sigma_{1} \tau-\psi_{1}\right) \\
- & \frac{j_{2}-j_{3}}{4}\left(2+\sigma_{2}\right) \omega_{z} a_{2} \cos \left(\psi_{1}-\psi_{2}\right)-\frac{1}{2} c_{1} a_{1} \\
& +\frac{j_{2}}{4} a_{1} a_{2}^{2} \sin \left(2\left(\psi_{1}-\psi_{2}\right)\right) \\
\dot{a}_{2}=- & \frac{1}{4}\left(j_{4}-1\right)\left(\sigma_{2}-2\right) \omega_{z} a_{1} \cos \left(\psi_{1}-\psi_{2}\right)-\frac{1}{2} c_{2} a_{2} \\
- & \frac{1}{8} j_{4} a_{1}^{2} a_{2} \sin \left(2\left(\psi_{1}-\psi_{2}\right)\right), \\
a_{1} \dot{\psi}_{1}= & -\frac{3}{8} \alpha_{1} a_{1}^{3}+\frac{4 j_{1}+\left(j_{2}-j_{3}\right)\left(j_{4}-1\right)}{8} \omega_{z}^{2} a_{1} \\
& +\frac{j_{2}}{4} a_{1} a_{2}^{2} \cos \left(2\left(\psi_{1}-\psi_{2}\right)\right)-\frac{f_{0}}{2} \sin \left(\sigma_{1} \tau-\psi_{1}\right) \\
& +\frac{j_{2}-j_{3}}{4}\left(2+\sigma_{2}\right) \omega_{z} a_{2} \sin \left(\psi_{1}-\psi_{2}\right) \\
a_{2} \dot{\psi}_{2}= & \sigma_{2} a_{2}-\frac{3}{8} \alpha_{2} a_{2}^{3}+\frac{\omega_{z}^{2}}{8}\left(j_{3}-j_{2}+\left(4+j_{2}-j_{3}\right) j_{4}\right) a_{2} \\
& +\frac{j_{4}}{8} a_{1}^{2} a_{2}\left(\cos \left(2\left(\psi_{1}-\psi_{2}\right)\right)-2\right) \\
& -\frac{j_{4}-1}{4}\left(\sigma_{2}-2\right) \omega_{z} a_{1} \sin \left(\psi_{1}-\psi_{2}\right)
\end{aligned}
$$

where $a_{1}=\varepsilon \tilde{a}_{1}, a_{2}=\varepsilon \tilde{a}_{2}$.

The initial conditions supplementing equations (50)-(53) are

$a_{1}(0)=a_{10}, \quad \psi_{1}(0)=\psi_{10}$,

$a_{2}(0)=a_{20}, \quad \psi_{2}(0)=\psi_{20}$,

where $a_{10}, a_{20}, \psi_{10}, \psi_{20}$ are that known quantities like that the initial conditions (11) and (54) are compatible each to other.

Rejection of the secular terms guarantee not only that solutions of vibration problem are bounded but also it gives a way to determine the unknown functions $B_{1}\left(\tau_{1}, \tau_{2}\right), B_{2}\left(\tau_{1}, \tau_{2}\right)$. It should be noticed that although differential equations (50)-(53) are written using derivatives with respect to the time $\tau$, they describe the evolution of the functions $a_{1}\left(\tau_{1}, \tau_{2}\right)$, $a_{1}\left(\tau_{1}, \tau_{2}\right), \quad \psi_{1}\left(\tau_{1}, \tau_{2}\right), \psi_{2}\left(\tau_{1}, \tau_{2}\right)$ with respect to the slow times variables $\tau_{1}$ and $\tau_{2}$ because these functions a priori depend only on the slow times variables.

After solving initial value problem (50)-(54), we apply inversely formula (49) what allow to express the complex-valued functions $B_{1}\left(\tau_{1}, \tau_{2}\right), B_{2}\left(\tau_{1}, \tau_{2}\right)$ and their complex conjugates through the real-valued functions $a_{1}\left(\tau_{1}, \tau_{2}\right), a_{1}\left(\tau_{1}, \tau_{2}\right), \quad \psi_{1}\left(\tau_{1}, \tau_{2}\right), \quad \psi_{2}\left(\tau_{1}, \tau_{2}\right)$ in solutions of the equations system (27)-(32). Next, taking into account Eq. (15) we can write the approximate solution of original problem (10)-(12) related to the simultaneously occurring main and internal resonances. The solution has the following analytical form

$$
\begin{aligned}
\varphi= & a_{1} \cos \left(\tau+\psi_{1}\right)-\frac{1}{32} \alpha_{1} a_{1}^{3} \cos \left(3 \tau+3 \psi_{1}\right) \\
& -\frac{1}{16} j_{2} a_{1} a_{2}^{2} \cos \left(3 \tau+\psi_{1}+2 \psi_{2}\right) \\
\vartheta= & a_{2} \cos \left(\tau+\psi_{2}\right)+\frac{1}{32} j_{4} a_{1}^{2} a_{2} \cos \left(3 \tau+2 \psi_{1}+\psi_{2}\right) \\
& -\frac{1}{32} \alpha_{2} a_{2}^{3} \cos \left(3 \tau+3 \psi_{2}\right) .
\end{aligned}
$$

The quantities $a_{1}, a_{2}$ and $\psi_{1}, \psi_{2}$ introduced into consideration by exponential representation (49) are functions of slow time variables and occur respectively as the amplitudes and the phases of the components of approximate solution (55)-(56). So, initial value problem (50)-(54) which is associated with basic equations set (27)-(32) determine the slow evolution of the vibration amplitudes and phases in time. For that reason, this issue is known as modulation problem. Contrary to the previously discussed case of the non-resonant vibration, initial value problem (50)-(54) cannot be solved in the analytical manner.

The time histories of the generalized coordinates in case of the doubled resonance are presented in Fig. 4. The values of parameters assumed in this simulation are as follows:

$$
\begin{aligned}
\sigma_{1} & =0.01, \quad \sigma_{2}=0.04167, \quad f_{0}=0.0043662, \quad \alpha_{1}=1, \\
\alpha_{2} & =1, \quad c_{1}=0.0000575531, \quad c_{2}=0.0000575531, \\
\omega_{z} & =0.0000575531, j_{1}=0.04, \quad j_{2}=0, \quad j_{3}=0.96, \quad j_{4}=0, \\
a_{10} & =0.008, \quad a_{20}=0.008, \quad \psi_{10}=0, \quad \psi_{20}=0 .
\end{aligned}
$$

In Fig. 4a, the black solid line represent the amplitude $a_{1}$ whereas the black line depicted in Fig. $4 \mathrm{~b}$ is the image of the amplitude $a_{2}$. Both curves envelop the graphs of fast changing oscillations. The nondimensional value $\tau_{\max }=20,000$ denoting the duration of simulation correspond to about $1.08 \mathrm{~s}$.

Similarly as in Sect. 4, in Fig. 4 are depicted the approximate solutions obtained using MSM and calculated numerically. The values of error (23) for the solutions derived using MSM are as follows

$\delta_{1}=1.400831 \cdot 10^{-7}, \quad \delta_{2}=8.920221 \cdot 10^{-9}$.

For comparison, the values of error for the solutions obtained by the optimized numerical method in Mathematica 11.1 are

$\delta_{1}=2.562360 \cdot 10^{-7}, \quad \delta_{2}=6.434821 \cdot 10^{-8}$.

Both values of error measuring satisfaction of the governing equations by approximate solution are smaller in case of MSM solution. 
Fig. 4 Time histories in case of doubled resonance: a the generalized coordinate $\varphi$ and amplitude $a_{1} ; \mathbf{b}$ the generalized coordinate $\vartheta$ and amplitude $a_{2}$

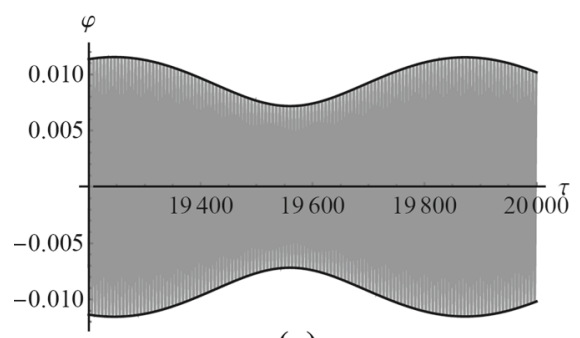

(a)

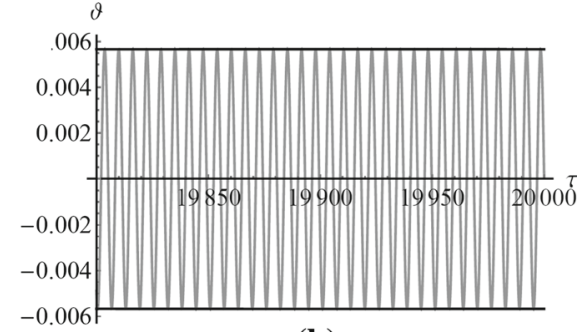

(b)

\section{Steady-state responses}

Initial value problem (50)-(54) arose in process of solving motion equations (10)-(12) using MSM is a good basis for studying the steady-state forced vibration of the micromechanical gyroscope. For this purpose, it is convenient to introduce modified phases $\Psi_{1}(\tau)$ and $\Psi_{2}(\tau)$ as follows

$\psi_{1}(\tau)=\sigma_{1} \tau-\Psi_{1}(\tau), \quad \psi_{2}(\tau)=\sigma_{1} \tau-\Psi_{2}(\tau)$.

Applying expressions (59) transforms modulation problem (50)-(54) into an autonomous form that is suitable to analyze steady-state motion of the system. Oscillations of forced system can achieve the steady state when all transient processes disappear. The symptom of this state is fixing of the values of the amplitudes $a_{1}, a_{2}$ and modified phases $\psi_{1}, \psi_{2}$. By zeroing of the derivatives of amplitudes and modified phases in modulation equations (50)-(53), we get the conditions of a steady state

$$
\begin{aligned}
& 2 f_{0} \cos \left(\Psi_{1}\right)+\left(j_{2}-j_{3}\right)\left(2+\sigma_{2}\right) \omega_{z} a_{2} \cos \left(\Psi_{1}-\Psi_{2}\right) \\
& \quad+a_{1}\left(2 c_{1}+j_{2} a_{2}^{2} \sin \left(2\left(\Psi_{1}-\Psi_{2}\right)\right)\right)=0 \\
& 2\left(j_{4}-1\right)\left(\sigma_{2}-2\right) \omega_{z} a_{1} \cos \left(\Psi_{1}-\Psi_{2}\right) \\
& \quad+4 a_{2} c_{2}-j_{4} a_{1}^{2} a_{2} \sin \left(2\left(\Psi_{1}-\Psi_{2}\right)\right)=0 \\
& -8 \sigma_{1} a_{1}-3 \alpha_{1} a_{1}^{3}+a_{1}\left(\left(4 j_{1}+\left(j_{2}-j_{3}\right)\left(j_{4}-1\right)\right) \omega_{z}^{2}\right. \\
& \quad+2 j_{2} a_{2}^{2} \cos \left(2\left(\Psi_{1}-\Psi_{2}\right)\right)-4 f_{0} \sin \left(\Psi_{1}\right) \\
& \quad-2\left(j_{2}-j_{3}\right)\left(2+\sigma_{2}\right) \omega_{z} a_{2} \sin \left(\Psi_{1}-\Psi_{2}\right)=0, \\
& -8 \sigma_{1} a_{2}+8 a_{2} \sigma_{2}-3 \alpha_{2} a_{2}^{3} \\
& \quad+a_{2}\left(j_{3}-j_{2}+\left(4+j_{2}-j_{3}\right) j_{4}\right) \omega_{z}^{2} \\
& \quad+j_{4} a_{1}^{2} a_{2}\left(\cos \left(2\left(\Psi_{1}-\Psi_{2}\right)\right)-2\right) \\
& \quad-2\left(j_{4}-1\right)\left(\sigma_{2}-2\right) \omega_{z} a_{1} \sin \left(\Psi_{1}-\Psi_{2}\right)=0 .
\end{aligned}
$$

The solution of system of nonlinear equations (60)(63) determine the values of amplitudes and modified phases of the gyroscope vibration in case of main and internal resonances that occur simultaneously.
Let us analyze resonant response of micromechanical gyroscope in the general case, i.e., without any additional assumptions about its properties. The following values of parameters are fixed

$$
\begin{aligned}
& \text { SET1 }=\left\{\sigma_{2}=-0.00333, \quad f_{0}=0.0000011,\right. \\
& \alpha_{1}=1.42, \quad \alpha_{2}=2.174, \quad c_{1}=0.000010127, \\
& c_{2}=0.00001, \quad \omega_{z}=0.000131656, \quad j_{1}=0.0769, \\
& \left.j_{2}=0, \quad j_{3}=0.923, \quad j_{4}=0\right\} .
\end{aligned}
$$

The value of the detuning parameter $\sigma_{1}$ is increased regularly by 0.000015 , starting from $\sigma_{1}=-0.0065$. The resonant response curves are obtained solving equations (60)-(63) with help the procedure NSolve offered in Mathematica 11.1. The results are presented in Fig. 5.

The resonant answers of the system exhibit high coincidence with the numerical solution of the motion equations. The simulation was carried out assuming the same values of parameter. Additionally, it is assumed

$$
\begin{aligned}
& \sigma_{1}=-0.004, \quad a_{10}=0.01, \quad a_{20}=0.01, \\
& \psi_{10}=0, \quad \psi_{20}=0 .
\end{aligned}
$$

Time histories of the generalized coordinates for this data are given in Fig. 6. Both graphs present the vibration just before the end of simulation which was realized for $\tau \in\left[0, \tau_{\max }\right]$, where $\tau_{\max }=20,000$ corresponds to about $1.32 \mathrm{~s}$.

The values of error (23) for the approximate solution obtained using MSM are as follows

$\delta_{1}=1.456467 \cdot 10^{-9}, \quad \delta_{2}=2.940916 \cdot 10^{-9}$,

while the values of the error for the numerical results obtained using Mathematica Software are

$\delta_{1}=2.289364 \cdot 10^{-7}, \quad \delta_{2}=2.316752 \cdot 10^{-7}$.

Equations (60)-(63) allow for a complete analysis of the gyroscope steady-state response for various special case studies. 
Fig. 5 Resonance curves: a for the gimbal vibration, $\mathbf{b}$ for the sensing element vibration; set of data: SET1

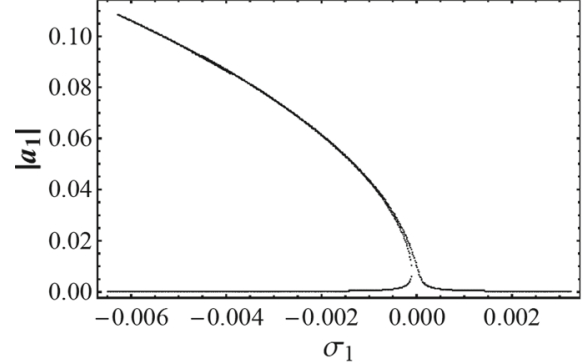

(a)

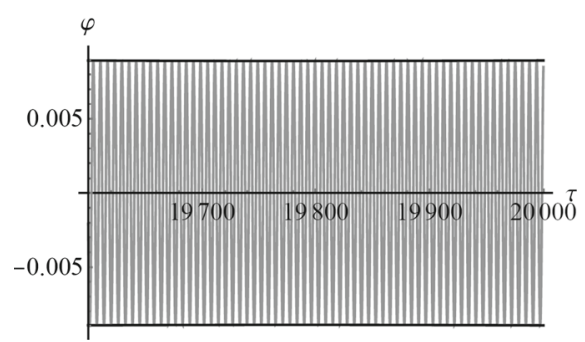

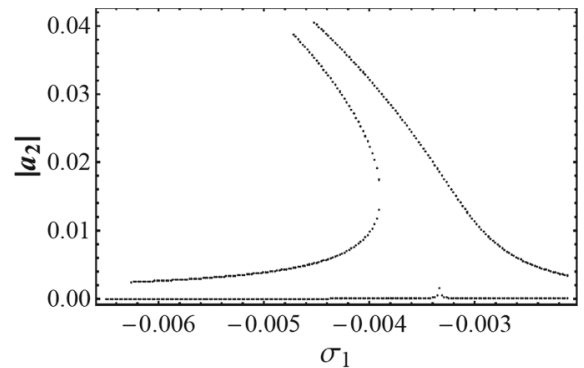

(b)

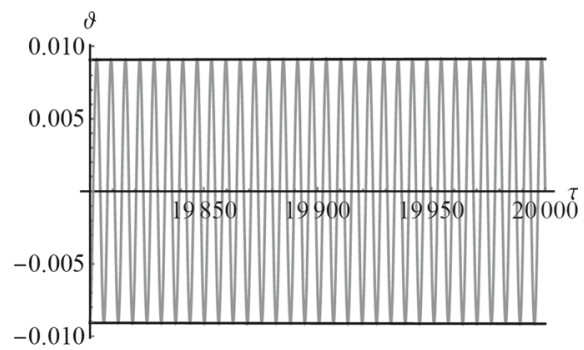

Fig. 6 Time histories of generalized coordinates for the set of data: SET1
Case $1\left(\alpha_{1}=0, \alpha_{2}=0\right)$

Let us consider the micromechanical gyroscope the torsional joints of which have strictly linear elastic characteristic. Inserting $\alpha_{1}=0, \quad \alpha_{2}=0$ into equations (60)-(63), one gets

$$
\begin{aligned}
& 2 f_{0} \cos \left(\Psi_{1}\right)+\left(j_{2}-j_{3}\right)\left(2+\sigma_{2}\right) \omega_{z} a_{2} \cos \left(\Psi_{1}-\Psi_{2}\right) \\
& \quad+a_{1}\left(2 c_{1}+j_{2} a_{2}^{2} \sin \left(2\left(\Psi_{1}-\Psi_{2}\right)\right)\right)=0 \\
& 2\left(j_{4}-1\right)\left(\sigma_{2}-2\right) \omega_{z} a_{1} \cos \left(\Psi_{1}-\Psi_{2}\right)+4 a_{2} c_{2} \\
& \quad-j_{4} a_{1}^{2} a_{2} \sin \left(2\left(\Psi_{1}-\Psi_{2}\right)\right)=0 \\
& -8 \sigma_{1} a_{1}+a_{1}\left(\left(4 j_{1}+\left(j_{2}-j_{3}\right)\left(j_{4}-1\right)\right) \omega_{z}^{2}\right. \\
& \quad+2 j_{2} a_{2}^{2} \cos \left(2\left(\Psi_{1}-\Psi_{2}\right)\right)-4 f_{0} \sin \left(\Psi_{1}\right) \\
& -2\left(j_{2}-j_{3}\right)\left(2+\sigma_{2}\right) \omega_{z} a_{2} \sin \left(\Psi_{1}-\Psi_{2}\right)=0 \\
& -8 \sigma_{1} a_{2}+8 a_{2} \sigma_{2}+a_{2}\left(j_{3}-j_{2}+\left(4+j_{2}-j_{3}\right) j_{4}\right) \omega_{z}^{2} \\
& \quad+j_{4} a_{1}^{2} a_{2}\left(\cos \left(2\left(\Psi_{1}-\Psi_{2}\right)\right)-2\right) \\
& \quad-2\left(j_{4}-1\right)\left(\sigma_{2}-2\right) \omega_{z} a_{1} \sin \left(\Psi_{1}-\Psi_{2}\right)=0
\end{aligned}
$$

Assumption about vanishing nonlinear does not cause any significant simplifications. The modulation equations are still nonlinear and majority of the nonlinear terms is conditioned by the inertial properties of the sensing plate.

Case $2\left(\alpha_{1}=0, \alpha_{2}=0, j_{2}=0, j_{4}=0\right)$ Observe that the steady-state equations become much simpler when $j_{2}=j_{4}=0$. These relations are satisfied if the components $J_{x}$ and $J_{z}$ of the diagonal inertia tensor $\hat{\mathbf{J}}$ of the sense plate are equal to each other. Modulation equations take the following form

$$
\begin{aligned}
& 2 f_{0} \cos \left(\Psi_{1}\right)-j_{3}\left(2+\sigma_{2}\right) \omega_{z} a_{2} \cos \left(\Psi_{1}-\Psi_{2}\right)+2 a_{1} c_{1}=0, \\
& 2\left(\sigma_{2}-2\right) \omega_{z} a_{1} \cos \left(\Psi_{1}-\Psi_{2}\right)-4 a_{2} c_{2}=0, \\
& -8 \sigma_{1} a_{1}+4 j_{1} \omega_{z}^{2} a_{1}+j_{3} \omega_{z}^{2} a_{1} \\
& \quad-4 f_{0} \sin \left(\Psi_{1}\right)+2 j_{3}\left(2+\sigma_{2}\right) \omega_{z} a_{2} \sin \left(\Psi_{1}-\Psi_{2}\right)=0, \\
& -8 \sigma_{1} a_{2}+a_{2}\left(8 \sigma_{2}+j_{3} \omega_{z}^{2}\right)-2\left(\sigma_{2}-2\right) \omega_{z} a_{1} \sin \left(\Psi_{1}-\Psi_{2}\right)=0 .
\end{aligned}
$$

The modified phases can be eliminated from equations (70)-(73) using trigonometric identities what allows to express explicitly the amplitude-frequency dependencies

$$
\begin{aligned}
& \frac{a_{2}^{2}\left(16 c_{2}^{2}+\left(8 \sigma_{2}-8 \sigma_{1}+j_{3} \omega_{z}^{2}\right)^{2}\right)}{4 a_{1}^{2}\left(\sigma_{2}-2\right)^{2} \omega_{z}^{2}}=1 \\
& \left(4 a_{1} j_{1} \omega_{z}^{2}-8 a_{1} \sigma_{1}+a_{1} j_{3} \omega_{z}^{2}+\frac{a_{2}^{2} j_{3}\left(2+\sigma_{2}\right)\left(8 \sigma_{2}-8 \sigma_{1}+j_{3} \omega_{z}^{2}\right)}{a_{1}\left(\sigma_{2}-2\right)}\right)^{2} \\
& +\frac{16\left(a_{1}^{2} c_{1}\left(\sigma_{2}-2\right)-a_{2}^{2} c_{2} j_{3}\left(2+\sigma_{2}\right)\right)^{2}}{a_{1}^{2}\left(\sigma_{2}-2\right)^{2}}=16 f_{0}^{2} .
\end{aligned}
$$

We get the system of two algebraic equations of the 8 -th order with respect to the unknown amplitudes $a_{1}$ and $a_{2}$.

The resonance curves obtained in result of solving system (74)-(75) for the following data of parameters

SET2 $=\left\{f_{0}=1 . \times 10^{-7}, \alpha_{1}=0, \alpha_{2}=0, c_{1}=\right.$ 5. $\times 10^{-7}, c_{2}=5 . \times 10^{-7}, \omega_{z}=3 . \times 10^{-6}, j_{1}=$ $\left.0.00826, j_{3}=0.9917\right\}$ are presented in Fig. 7 . 


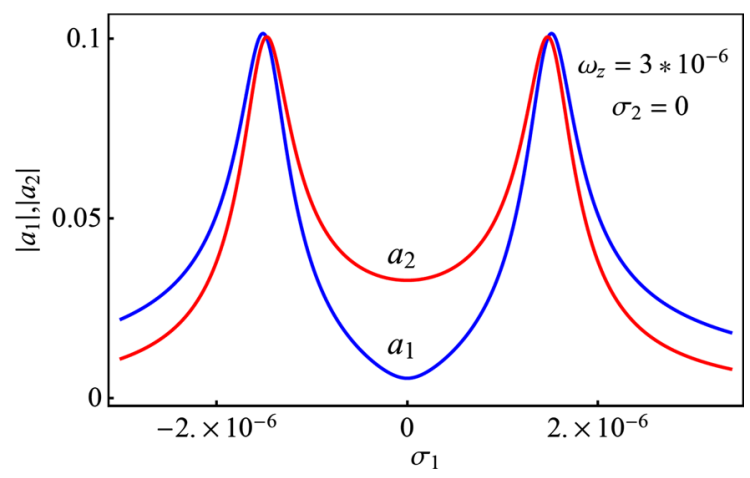

Fig. 7 Resonance curves; $a_{1}$-amplitude of the gimbal, $a_{2}$ amplitude of the sensing plate

The full symmetry of the graphs depicted in Fig. 7 is typical behavior of the considered two-degrees-offreedom linear system.

The mentioned symmetry is disturbed when the system is not perfectly tuned to the internal resonance, i.e., when $\sigma_{2} \neq 0$. The angular velocity of the substrate has also crucial influence on the resonant response. The influence on the resonant response of the detuning parameter $\sigma_{2} \neq 0$, which means that the system is not perfectly tuned, and with respect to the angular velocity $\omega_{z}$ is presented in Fig. 8.

Comparing several cases presented in Figs. 7 and 8, we can observe that resonant picks are moving away from each other when $\omega_{z}$ increases. However, the increase of $\sigma_{2}$ disturbs symmetry of the graphs.

Case $3\left(j_{1}=j_{2}=j_{4}=0, j_{3}=1\right)$

In this case, we assume that moments of inertia of the gimbal are assumed as negligible and that the tensor of inertia of the sensor element is isotropic. Inserting the assumptions $j_{1}=j_{2}=j_{4}=0, j_{3}=1$ into modulation equations (60)-(63), we get

$2 f_{0} \cos \left(\Psi_{1}\right)+\left(2+\sigma_{2}\right) \omega_{z} a_{2} \cos \left(\Psi_{1}-\Psi_{2}\right)+2 a_{1} c_{1}=0$,

$2\left(\sigma_{2}-2\right) \omega_{z} a_{1} \cos \left(\Psi_{1}-\Psi_{2}\right)-4 a_{2} c_{2}=0$,

$-8 a_{1} \sigma_{1}-3 \alpha_{1} a_{1}^{3}+a_{1} \omega_{z}^{2}$

$-4 f_{0} \sin \left(\Psi_{1}\right)+2\left(2+\sigma_{2}\right) \omega_{z} a_{2} \sin \left(\Psi_{1}-\Psi_{2}\right)=0$,

$-8 a_{2} \sigma_{1}+a_{2}\left(-3 \alpha_{2} a_{2}^{2}+8 \sigma_{2}+\omega_{z}^{2}\right)$

$-2\left(\sigma_{2}-2\right) \omega_{z} a_{1} \sin \left(\Psi_{1}-\Psi_{2}\right)=0$.

Considered here assumptions cause vanishing of trigonometric functions whose argument is difference of the modified phases multiplied by two. This circumstance allow to eliminate from (76)-(79) the other trigonometric functions of the modified phases. In this manner, the following implicit dependence between the amplitudes and frequencies in the resonance zone is obtained

$$
\begin{aligned}
& \frac{a_{2}^{2}\left(16 c_{2}^{2}+\left(-3 \alpha_{2} a_{2}^{2}+8 \sigma_{2}-8 \sigma_{1}+\omega_{z}^{2}\right)^{2}\right)}{4 a_{1}^{2}\left(\sigma_{2}-2\right)^{2} \omega_{z}^{2}}=1, \\
& \left(-3 \alpha_{1} a_{1}^{3}-8 \sigma_{1} a_{1}+\omega_{z}^{2} a_{1}+\frac{a_{2}^{2}\left(2+\sigma_{2}\right)\left(-3 \alpha_{2} a_{2}^{3}+8 \sigma_{2}-8 \sigma_{1}+\omega_{z}^{2}\right)}{a_{1}\left(\sigma_{2}-2\right)}\right)^{2} \\
& +\frac{16\left(c_{1}\left(\sigma_{2}-2\right) a_{1}^{2}-c_{2}\left(2+\sigma_{2}\right) a_{2}^{2}\right)^{2}}{a_{1}^{2}\left(\sigma_{2}-2\right)^{2}}=16 f_{0}^{2} .
\end{aligned}
$$

Equations (80)-(81) form the algebraic set of 48-th order with respect to the unknown amplitudes $a_{1}$ and $a_{2}$, where amplitudes appear only in even powers. These equations allow to perform qualitative analysis of the resonance steady-state amplitudes versus detuning parameter $\sigma_{1}$ for various parameters.

The analysis of the influence of the damping coefficient on the amplitudes is presented in Fig. 9. The assumed values of system parameters are:

$$
\begin{aligned}
\mathrm{SET} 3= & \left\{f_{0}=4.3662 \times 10^{-7}, \quad \alpha_{1}=1,\right. \\
& \left.\alpha_{2}=1, \quad \omega_{z}=6 . \times 10^{-5}, \quad \sigma_{2}=0\right\}
\end{aligned}
$$

The influence of the amplitude $f_{0}$ on the resonance curves, for the set of parameters SET3 and $c_{1}=c_{2}=$ $4 \times 10^{-5}$, is presented in Fig. 10 .

The nonlinearity parameters $\alpha_{1}$ and $\alpha_{2}$ have also essential impact on the resonant characteristics for the set of parameters SET3 and $c_{1}=c_{2}=2.5 \times 10^{-6}$, is presented in Fig. 11.

The values of damping coefficients for which the resonance curves become unique can be estimated, in the way of numerical simulations, for the given microsystem and given loading. In the case of data values specified in SET3, coefficients $c_{1}=c_{2}=0.000034$ fulfill this criterion.

Case $4\left(\omega_{z}=0\right)$

In the case of immovable substrate the form of modulation equations is simplified to the following

$$
\begin{aligned}
& \left.2 f_{0} \cos \left(\Psi_{1}\right)+2 a_{1} c_{1}-j_{2} a_{1} a_{2}^{2} \sin \left(2\left(\Psi_{2}-\Psi_{1}\right)\right)\right)=0, \\
& 4 a_{2} c_{2}-j_{4} a_{2} a_{1}^{2} \sin \left(2\left(\Psi_{1}-\Psi_{2}\right)\right)=0, \\
& -8 a_{1} \sigma_{1}-3 \alpha_{1} a_{1}^{3}+2 j_{2} a_{1} a_{2}^{2} \cos \left(2\left(\Psi_{1}-\Psi_{2}\right)\right) \\
& \quad-4 f_{0} \sin \left(\Psi_{1}\right)=0, \\
& -8 a_{2} \sigma_{1}+8 a_{2} \sigma_{2}-3 \alpha_{2} a_{2}^{3} \\
& \quad-2 a_{1}^{2} a_{2} j_{4}+a_{1}^{2} a_{2} j_{4} \cos \left(2\left(\Psi_{1}-\Psi_{2}\right)\right)=0 .
\end{aligned}
$$

The inertial parameters $j_{1}$ and $j_{3}$ do not appear in equations (82)-(85). The trigonometric identities again allow to eliminate the functions $\Psi_{1}$ and $\Psi_{2}$. 

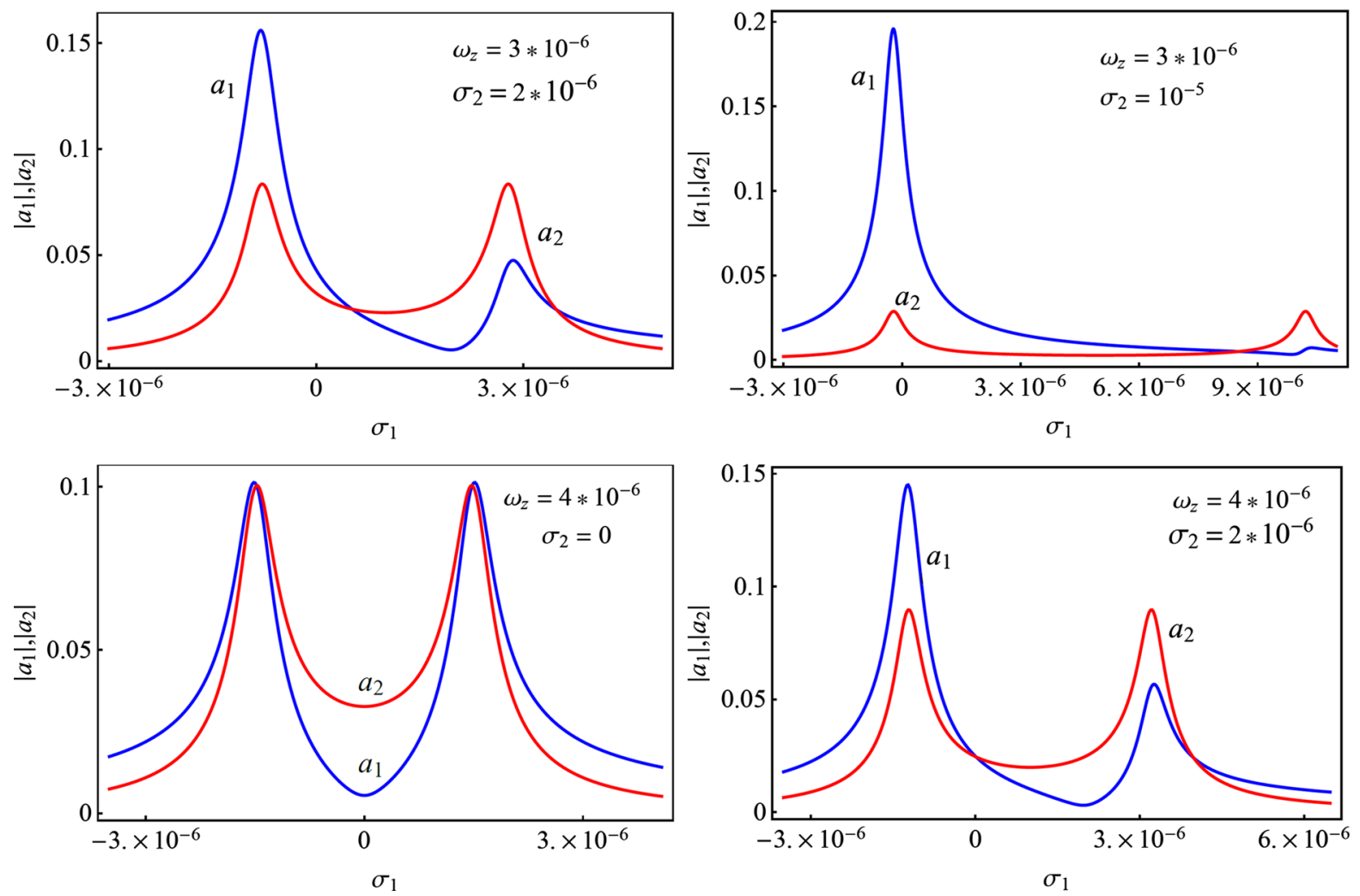

Fig. 8 Resonance curves for various $\omega_{z}$ and $\sigma_{2}$ (set of data: SET2)
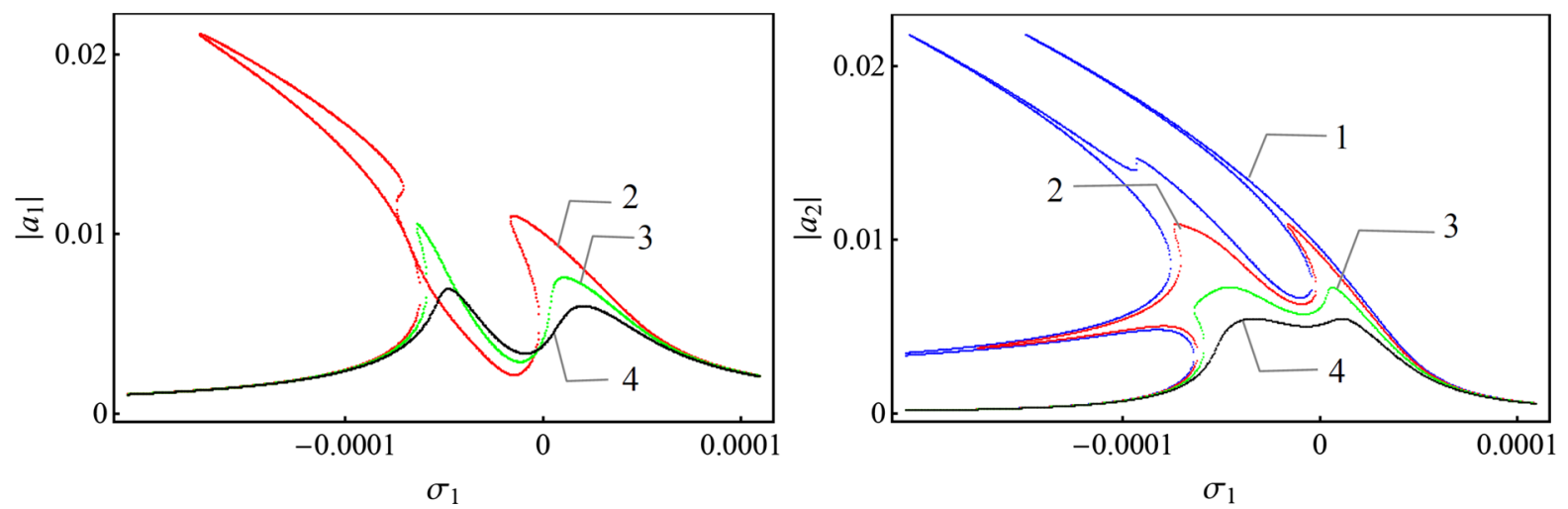

Fig. 9 Resonance curves for: (1) $c_{1}=c_{2}=1 \times 10^{-5}$, (2) $c_{1}=c_{2}=2 \times 10^{-5}$, (3) $c_{1}=c_{2}=3 \times 10^{-5}$, (4) $c_{1}=c_{2}=4 \times 10^{-5}$

Case $5\left(\omega_{z}=0, j_{2}=0, j_{4}=0\right)$

Let us assume that the substrate is immovable and additionally $J_{x}=J_{z}$, so $j_{2}=j_{4}=0$. The last assumption together with $\omega_{z}=0$ leads to the conclusion that $a_{2}=0$. When the substrate is at rest, then the sensing plate does not oscillate. The forced vibration of the gimbal does not excite the sensor what confirms that the Coriolis torque is the main reason causing the sense plate to vibrate. Due to the substrate and the sensor plate are at rest, the equation describing the relationship amplitude-frequency for gimbal is completely 

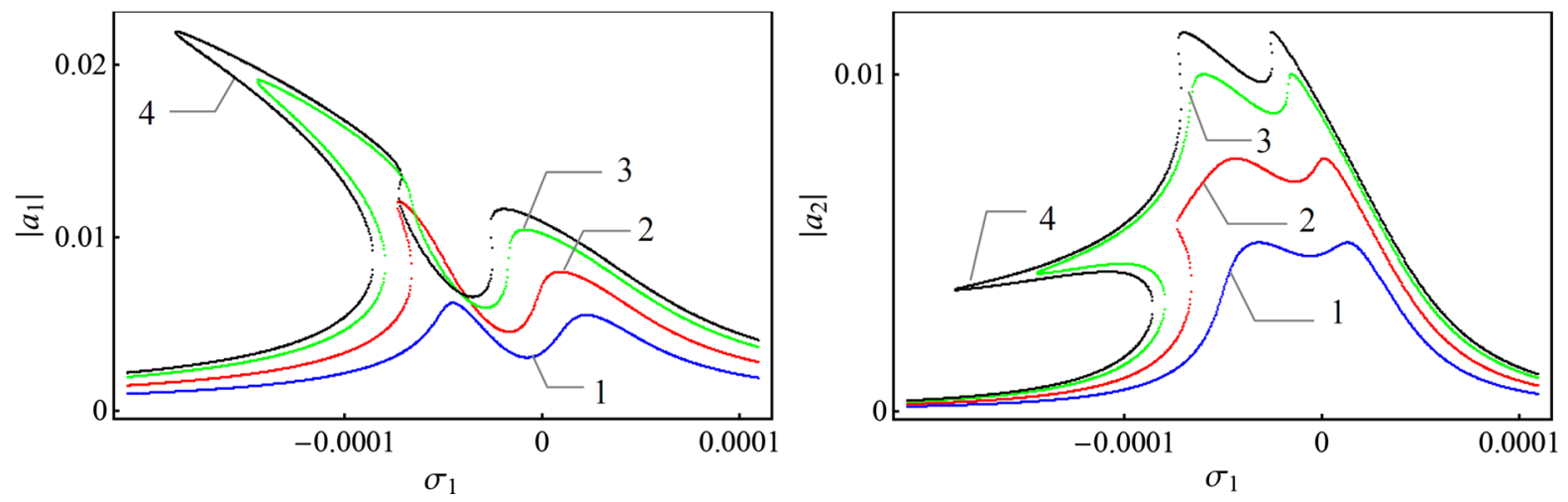

Fig. 10 Resonance curves for: (1) $f_{0}=4 . \times 10^{-7}$, (2) $f_{0}=6 . \times 10^{-7}$, (3) $f_{0}=8 . \times 10^{-7}$, (4) $f_{0}=9 . \times 10^{-7}$
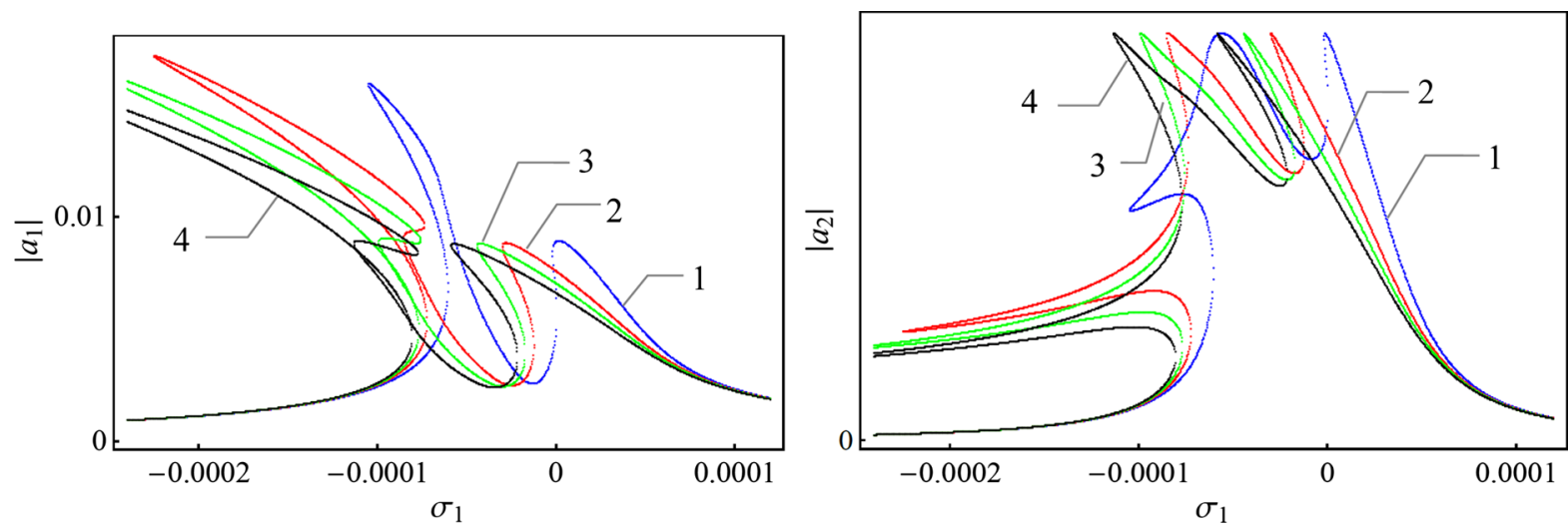

Fig. 11 Resonance curves for: (1) $\alpha_{1}=\alpha_{2}=1$, (2) $\alpha_{1}=\alpha_{2}=2$, (3) $\alpha_{1}=\alpha_{2}=2.5$, (4) $\alpha_{1}=\alpha_{2}=3$

uncoupled and has the following form

$a_{1}^{2}\left(16 c_{1}^{2}+\left(3 \alpha_{1} a_{1}^{2}+8 \sigma_{1}\right)^{2}\right)=16 f_{0}^{2}$.

The family of resonance curves, obtained using Eq. (86), for various values of damping coefficient $c_{1}$ is presented in Fig. 12. The values of parameters assumed in this simulation are

$$
\begin{aligned}
& \text { SET4 }=\left\{\sigma_{2}=-0.0394, \quad f_{0}=4 . \times 10^{-6},\right. \\
& \alpha_{1}=10, \quad \alpha_{2}=10, \quad c_{2}=0.000114, \\
& \left.j_{2}=0, \quad j_{4}=0\right\}
\end{aligned}
$$

Case 6 (identification of angular velocity)

The identification problem of substrate angular velocity requires an unambiguous character of each of the both resonance response curves. So, determination of the value of the damping coefficient which guarantee this unambiguity for given micromechanical gyroscope is of significant importance. It is also important to

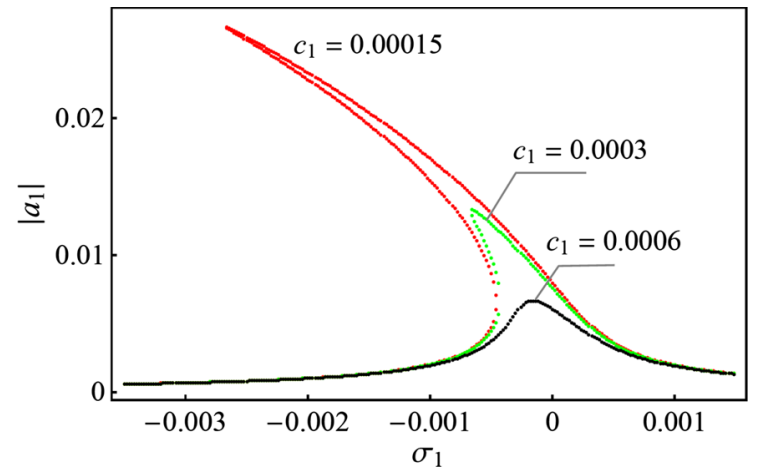

Fig. 12 Resonance curves for the gimbal when substrate is immovable

reduce the number of the measurement system parameters that can have influence on the identification. One of the earlier discussed cases leads to essential simplification of the equations of steady state. It is the case when the gimbal moments of inertia are sufficiently 

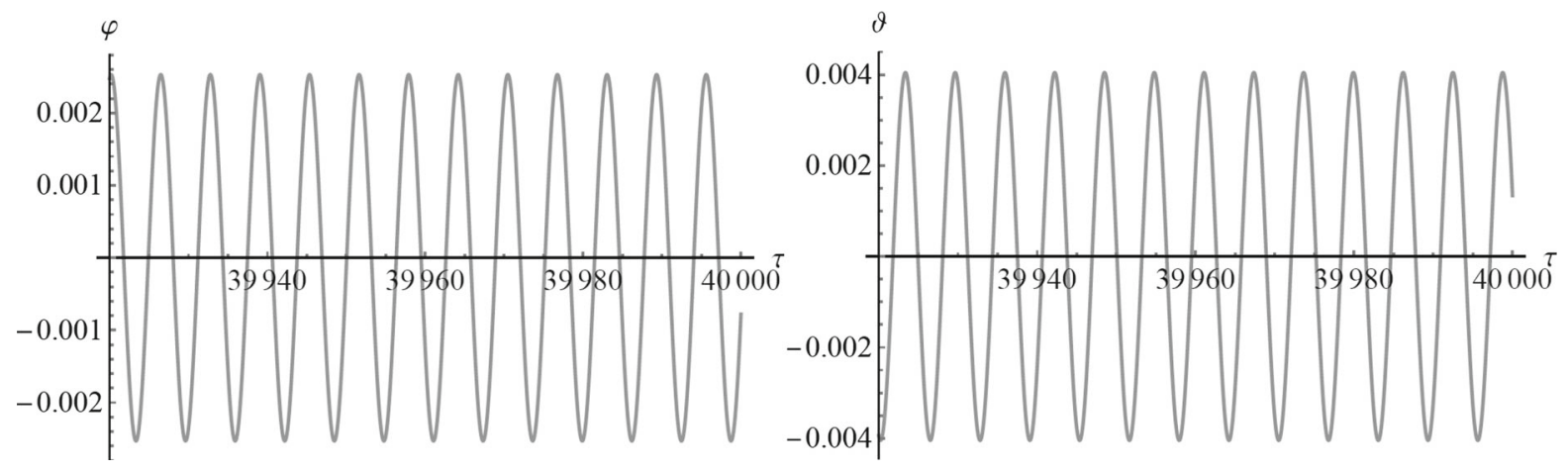

Fig. 13 Steady state vibration for the gimbal and the sensing plate
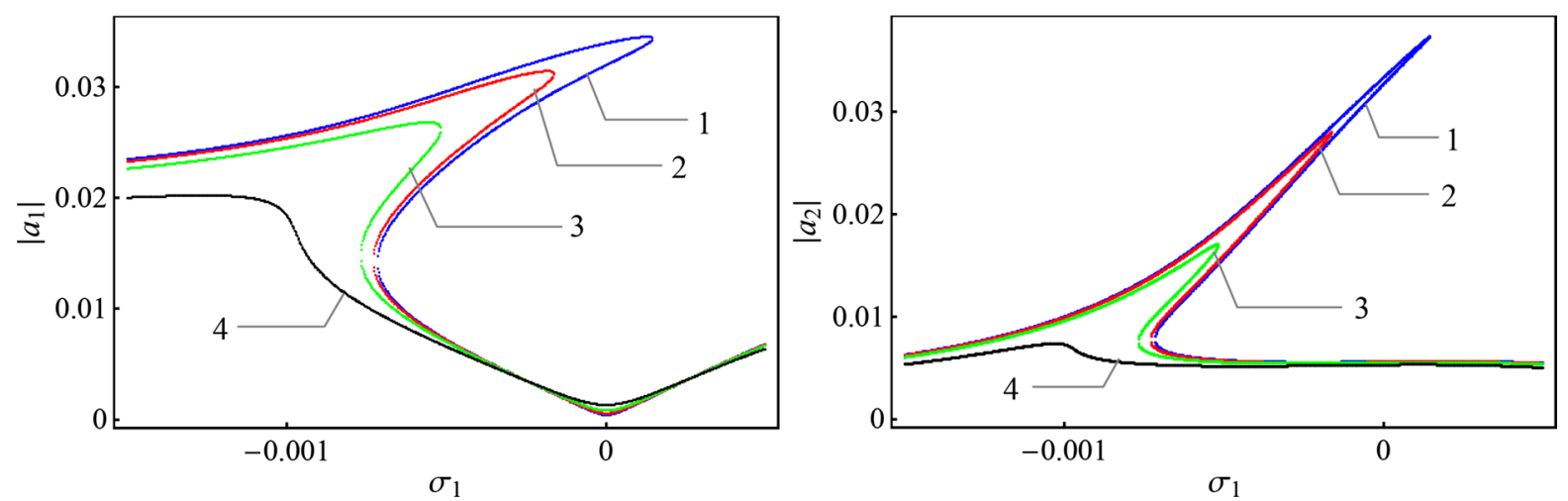

Fig. 14 Resonance curves. The amplitudes of the gimbal and the sensing plate versus $\sigma_{2}$ for various values of the damping coefficients; (1) $c_{1}=c_{2}=6 \times 10^{-5}$, (2) $c_{1}=c_{2}=8 \times 10^{-5}$, (3) $c_{1}=c_{2}=12 \times 10^{-5}$, (4) $c_{1}=c_{2}=3 \times 10^{-5}$, (4) $c_{1}=c_{2}=20 \times 10^{-5}$

slight and the tensor of inertia of the sense plate is isotropic. These assumptions that simplify the steady state equations to form (76)-(79) can be written as follows $j_{1}=j_{2}=j_{4}=0, j_{3}=1$. Equation (77) of this system is relatively simple and contains the smallest number of gyroscope parameters. Let us solve Eq. (77) for the angular velocity

$\omega_{z}=\frac{2 a_{2} c_{2}}{\left(\sigma_{2}-2\right) a_{1} \cos \left(\Psi_{1}-\Psi_{2}\right)}$.

When the system is perfectly designed, made and tuned, the detuning parameter $\sigma_{2}$ is equal to zero. Thus it is enough to know the damping coefficient $c_{2}$ and to measure the values of amplitudes $a_{1}$ and $a_{2}$ in the resonance. In the steady state, the difference of the modified phases should be set at value $\pi$. Assuming all these circumstances, we can write

$\omega_{z}=\frac{a_{2} c_{2}}{a_{1}}$.
The following simulation is carried out in order to apply this identification way. We assume some values of parameters of gyroscope including the value of the substrate angular velocity, namely

SET5 $=\left\{\sigma_{1}=0, \quad \sigma_{2}=0, \quad f_{0}=4.5 \times 10^{-6}\right.$, $\alpha_{1}=1, \alpha_{2}=1, \quad c_{1}=0.0005, \quad c_{2}=0.0005$, $\omega_{z}=0.0008, \quad a_{10}=0.005, \quad a_{20}=0.005$, $\left.\psi_{10}=0, \quad \psi_{10}=0\right\}$.

Then, we find the approximate solution of initial value problem (10)-(12) and determine the both values of amplitudes, replacing real measurement by the reading the values from the graphs. The variability over time of the both generalized coordinates for the steadystate vibration is presented in Fig. 13. Using graphs, we determine

$a_{1} \approx 0.000249, a_{2} \approx 0.004$, and hence $\omega_{z}$ $\approx 0.0008032$.

However, this procedure fails when due to any reason the assumptions concerning the coincidence of both 
resonances at the same resonant frequency $\left(\sigma_{2}=0\right)$ are not strictly satisfied. The higher value the parameter $\sigma_{2}$ takes, the less accurate the angular velocity measurement is. In Fig. 14, there is shown the influence of parameter $\sigma_{2}$ on the vibration amplitude of the both the gimbal and the sensing plate. This influence is especially spectacular when the damping is small and the resonance response curves are ambiguous. However, even for sufficiently large values of damping coefficients which make sure the curves are functions of the detuning parameter $\sigma_{2}$, the values of the both amplitudes change significantly with $\sigma_{2}$. The numerical simulation was carried out assuming the following fixed values of parameters

SET6 $=\left\{\sigma_{1}=-10^{-5}, \quad f_{0}=4.5 \times 10^{-6}\right.$, $\left.\alpha_{1}=1, \quad \alpha_{2}=1, \quad \omega_{z}=0.0008\right\}$.

\section{Concluding remarks}

The equations of motion of micromechanical gyroscope of torsional type has been derived. The mathematical model includes nonlinear characteristics of the torsional links. According to actual work regime of this type micromechanical gyroscope, only harmonically changing torque acting about the gimbal axis has been assumed. The dynamical problem has been solved using the method of multiple time scales belonging to wide class of asymptotic methods. The significant advantage of the asymptotic methods consist in the analytical form of the approximate solutions. That gives the opportunity to both qualitative and quantitative analysis of the behavior of the system for wide range of its parameters.

The approximate solution of analytical form has been obtained firstly for non-resonant vibration what among other allow to detect the resonance conditions. The main and internal resonances simultaneously occurring have been the subject of our study. The characteristic feature of this type gyroscope is that the both resonance frequencies are equal to each other. The approximate solution has been obtained also for the resonant vibration. Initial value problem of the modulation strictly associated with the procedure of solving the governing equations using MSM gives the possibility to analyze the slow evolution of the amplitudes and phases.

Steady-state resonance oscillations are desired regime of work of the micromechanical gyroscope, so this situation has been analyzed in more detail. Moreover, some special cases have been investigated. For example, we simulated negligible inertia of gimbal and isotropic tensor of inertia of the sensor. Another case refers to situation when the torsional joints have strictly linear elastic characteristic, for both symmetric and non-symmetric moments of inertia. We have also shown that if substrate is immovable, the modulation equations are uncoupled and reduce to one equation describing vibration of a gimbal. In that case the sensing plate is motionless.

For all cases, the amplitude-frequency relationships have been derived. Resonance curves have been drawn illustrating influence of various parameters on their shape and uniqueness. Also an impact of damping coefficients and amplitude of the external excitation has been discussed.

The approximate analytical solution has been validated using the proposed measure of the error of fulfillment of the governing equations and also by comparison with the numerical simulation.

Acknowledgements This paper was financially supported by the grant of the Ministry of Science and Higher Education in Poland realized in Institute of Applied Mechanics of Poznan University of Technology (DS-PB: 02/21/DSPB/3513).

\section{Compliance with ethical standards}

Conflict of interest The authors declare that they have no conflict of interest.

Open Access This article is distributed under the terms of the Creative Commons Attribution 4.0 International License (http:// creativecommons.org/licenses/by/4.0/), which permits unrestricted use, distribution, and reproduction in any medium, provided you give appropriate credit to the original author(s) and the source, provide a link to the Creative Commons license, and indicate if changes were made.

\section{References}

1. Williams, C.B., Shearwood, C., Mellor, P.H., Mattingley, A.D., Gibbs, M.R., Yates, R.B.: Initial fabrication of a micro-induction gyroscope. Microelectron. Eng. 30, 531534 (1996)

2. Jin, L., Zhang, H., Zhong, Z.: Design of a lc-tuned magnetically suspended rotating gyroscope. J. Appl. Phys. 109, 07E525-07E525-3 (2011)

3. Turner, K.L., Miller, S.A., Hartwell, P.G., MacDonald, N.C., Strogatz, S.H., Adams, S.G.: Five parametric resonances in a micromechanical system. Nature 396, 149-152 (1998)

4. Lifshitz, R., Cross, M.C.: Response of parametrically driven nonlinear coupled oscillators with application to microme- 
chanical and nanomechanical resonator arrays. Phys. Rev. B 67, 1343021 (2003)

5. Gallacher, B.J., Burdess, J.S., Harish, K.M.: A control scheme for a MEMS electrostatic resonant gyroscope excited using combined parametric excitation and harmonic forcing. J. Micromech. Microeng. 16, 320-331 (2006)

6. Nayfeh, A.H., Younis, M.I.: Dynamics of MEMS resonators under superharmonic and subharmonic excitations. J. Micromech. Microeng. 15, 1840-1847 (2005)

7. Kacem, N., Hentz, S., Pinto, D., Reig, B., Nguyen, V.: Nonlinear dynamics of nanomechanical beam resonators: improving the performance of NEMS-based sensors. Nanotechnology 20, 275501 (2009)

8. Lestev, M.A., Tikhonov, A.A.: Nonlinear phenomena in the dynamics of micromechanical gyroscopes. Vest. St. Petersburg Univ. Math. 42(1), 53-57 (2009)

9. Zhang, W.-M., Meng, G., Wei, K.-X.: Dynamics of nonlinear coupled electrostatic micromechanical resonators under two-frequency parametric and external excitations. Shock Vib. 17, 759-770 (2010)

10. Martynenko, YuG, Mekuriev, I.V., Podalkov, V.V.: Dynamics of a ring micromechanical gyroscope in the forcedoscillation mode. Gyroscopy Navig. 1(1), 43-51 (2010)

11. Yoon, Sang Won, Lee, Sangwoo, Najafi, Khalil: Vibration sensitivity analysis of MEMS vibratory ring gyroscopes. Sens. Actuator A 171, 163-177 (2011)

12. Matheny, M.H., Villanueva, L.G., Karabalin, R.B., Sader, J.E., Roukes, M.L.: Nonlinear mode-coupling in nanomechanical systems. Nano Lett. 13, 1622-1626 (2013)

13. Ovchinnikova, N., Panferov, A., Ponomarev, V., Severov, L.: Control of vibrations in a micromechanical gyroscope using inertia properties of standing elastic waves. In: Proceedings of the $19^{\text {th }}$ World Congress, The International Federation of Automatic Control, Cape Town, South Africa, August 24-29, pp. 2679-2682 (2014)

14. Yoon, S., Park, U., Rhim, J., Yang, S.S.: Tactical grade MEMS vibrating ring gyroscope with high shock reliability. Microelectron. Eng. 142, 22-29 (2015)

15. Lestev, A.M.: Combination resonances in MEMs gyro dynamics. Gyroscopy Navig. 6(1), 41-44 (2015)
16. Nitzan, S.H., Zega, V., Li, M., Ahn, C.H., Corigliano, A., Kenny, T.W., Horsley, D.A.: Self-induced parametric amplification arising from nonlinear elastic coupling in a micromechanical resonating disk gyroscope. Sci. Rep. 5, 9036 (2015)

17. Defoort, M., Taheri-Tehrani, P., Nitzan, S., Horsley, D.A.: Synchronization in micromechanical gyroscopes. In: SolidState Sensors, Actuators and Microsystems Workshop, Hilton Head Island, South Carolina June 5-9, pp. 84-88 (2016)

18. Nitzan, S.H., Taheri-Tehrani, P., Defoort, M., Sonmezoglu, S.: Countering the effects of nonlinearity in rate-integrating gyroscopes. IEEE Sens. J. 16(10), 3556-3558 (2016)

19. Passaro, V.M.N., Cuccovillo, A., Vaiani, L., De Carlo, M., Campanella, C.E.: Gyroscope technology and applications: a review in the industrial perspective. Sensors 17, 22842288 (2017)

20. Starosta, R., Sypniewska-Kamińska, G., Awrejcewicz, J.: Nonlinear effects in dynamics of micromechanical gyroscope. Engineering dynamics and life sciences: DSTA 2017, red. J. Awrejcewicz, Department of Automation, Biomechanics and Mechatronics, 2017 - s. 511-520 (2017)

21. Awrejcewicz, J., Krysko, V.A.: Introduction to Asymptotic Methods. Chapman and Hall, Boca Raton (2006)

22. Shivamoggi, B.K.: Perturbation Methods for Differential Equations. Birkhauser, Boston (2003)

23. Starosta, R., Sypniewska-Kamińska, G., Awrejcewicz, J.: Asymptotic analysis of kinematically excited dynamical systems near resonances. Nonlinear Dyn. 68(4), 459-469 (2012)

24. Sypniewska-Kamińska, G., Starosta, R., Awrejcewicz, J.: Nonlinear vibration of rotating system near resonance. In: MATEC Web of Conferences, vol. 83 (2016)

25. Merkuriev, I.W., Podalkov, W.W.: Study of nonlinear dynamics of a micromechanical gyroscope. In: Proceedings of the IV International School-NDM "Nonlinear Dynamics of Machines", IMASH RAS, Moscow, pp. 82-91 (in Russian) (2017) 